\title{
Assessment of Comprehensive Analysis Calculation of Airloads on Helicopter Rotors
}

\author{
Hyeonsoo Yeo \\ Raytheon ITSS \\ NASA Ames Research Center \\ Moffett Field, California
}

\author{
Wayne Johnson \\ Army/NASA Rotorcraft Division \\ NASA Ames Research Center \\ Moffett Field, California
}

\begin{abstract}
Blade section normal force and pitching moment were investigated for six rotors operating at transition and high speeds: H-34 in flight and wind tunnel, SA 330 (research Puma), SA 349/2, UH-60A full-scale and BO105 model (HART-I). The measured data from flight and wind tunnel tests were compared with calculations obtained using the comprehensive analysis CAMRAD II. The calculations were made using two free wake models: rolled-up and multiple-trailer with consolidation models. At transition speed, there is fair to good agreement for the blade section normal force between the test data and analysis for the H-34, research Puma, and SA 349/2 with the rolled-up wake. The calculated airloads differ significantly from the measurements for the UH-60A and BO-105. Better correlation is obtained for the UH-60A and BO-105 by using the multiple-trailer with consolidation wake model. In the high speed condition, the analysis shows generally good agreement with the research Puma flight data in both magnitude and phase. However, poor agreement is obtained for the other rotors examined. The analysis shows that the aerodynamic tip design (chord length and quarter chord location) of the Puma has an important influence on the phase correlation.
\end{abstract}

\section{Notation}

$C_{T} \quad$ rotor thrust coefficient

G strength of trailed vorticity

M Mach number

$N_{b} \quad$ number of blades

$\mathrm{r} \quad$ blade radial station

$r_{C} \quad$ centroid of vorticity

$r_{G} \quad$ moment (radius of gyration) of vorticity

$\mathrm{R} \quad$ blade radius

Re Reynolds number

$\alpha_{T P P} \quad$ tip path plane tilt angle, positive forward

$\Gamma \quad$ bound circulation

$\mu \quad$ advance ratio

$\sigma \quad$ solidity

\section{Introduction}

The accurate prediction of rotor loads and vibration remains a difficult problem for helicopter design. Typically, helicopters encounter high vibration in two different speed regimes: transition and high speeds.

\footnotetext{
Presented at the American Helicopter Society 4th Decennial Specialist's Conference on Aeromechanics, San Francisco, California, January 21-23, 2004. This paper is declared a work of the U.S. Government and is not subject to copyright protection in the United States.
}

The rotor blade aerodynamic environment at transition speed is characterized by blade-vortex interactions, and at high speed by compressibility and a poor distribution of loading over the rotor disk. The ability to accurately predict the rotor aerodynamic loading for these two flight regimes is essential for the design of future rotorcraft.

For the past several decades a number of flight and wind tunnel tests have been conducted with pressureinstrumented blades, with the objective of improving the understanding of the nature of the aerodynamic loading acting on the rotor blade [1-6]. These extensive flight and wind tunnel data sets provide a valuable resource that can be used to evaluate the accuracy and reliability of comprehensive codes in airload prediction and to develop better methodologies to simulate the rotor aerodynamic environment.

Analyses need to provide consistently good predictions for various rotors and flight conditions in order to be used for design work. For applications to advanced configurations, the analyses must be based as much as possible on first-principle aerodynamics, minimizing the use of empirical models. The first step to obtain better prediction is to understand the deficiencies of the current analyses. This can be achieved by comparing calculations with flight and wind tunnel measurements for different rotors. Once the deficiencies are identified, effort can be made to improve the correlation. 
The purpose of this study is threefold: 1) assess the accuracy and reliability of a comprehensive code in the calculation of airloads, 2) understand the similarities and differences in blade airloads among the measurements as well as calculations and investigate the deficiencies of the current analysis and key elements to improve the correlation, and 3) carry out calculations with various wake models and identify important wake parameters for the airload calculation.

The calculations were performed using the comprehensive analysis CAMRAD II [7].

\section{Test Data}

Flight and wind tunnel test cases from various rotors [1-6] have been carefully selected so that they represent similar test conditions. Figure 1 and Table 1 show the thrust and advance ratio considered and some of the rotor parameters. The thrust coefficient values range from $C_{T} / \sigma=0.057$ to 0.079 , and the advance ratios are in the range of $\mu=0.129$ to 0.15 at transition, and $\mu=0.361$ to 0.39 at high speed.

The flight [1] and wind tunnel [2] tests of an H-34 helicopter have long been a standard for rotor airloads data. The section airloads data for the $\mathrm{H}-34$ in flight were measured at seven radial stations $(\mathrm{r} / \mathrm{R}=0.25,0.40,0.55$, $0.75,0.85,0.9$, and 0.95 ) and were averaged over three consecutive revolutions. The section airloads data for the $\mathrm{H}-34$ in wind tunnel were measured at nine radial stations $(\mathrm{r} / \mathrm{R}=0.25,0.40,0.55,0.75,0.85,0.9,0.95,0.97$, and $0.99)$ and were averaged over ten revolutions.

The research Puma (SA 330) data were obtained using a modified swept-tip blade rather than the standard rectangular Puma blade [3]. Absolute pressures were measured at $\mathrm{r} / \mathrm{R}=0.92,0.95$, and 0.978. A single revolution of data was taken for each test point, therefore, there is no averaging of data.

The SA 349/2 flight data were obtained from an upgraded Gazelle helicopter with three research Grande Vitesse (GV) blades [4]. Absolute pressures were measured at $\mathrm{r} / \mathrm{R}=0.75,0.88$, and 0.97 . Data from each of the pressure transducers were acquired over a period of six consecutive rotor revolutions and then averaged.

Test data with the UH-60A were obtained from the NASA/Army UH-60A Airloads Program conducted from August 1993 to February 1994 [5]. Absolute pressures were measured at $\mathrm{r} / \mathrm{R}=0.225,0.40,0.55,0.675,0.775$, $0.865,0.92,0.965$, and 0.99 . For this study, a single revolution of data was taken for each test point.

The BO-105 data were obtained from the Higherharmonic Acoustics Rotor Test (HART-I) program using a 40\% Mach-scaled hingeless BO-105 main rotor blade [6]. The objective of the test was to demonstrate the reduction of blade-vortex interactions (BVI) noise in the descending flights using higher-harmonic controls (HHC). The data set used for the current study is a baseline case without higher-harmonic pitch control inputs. A shaft angle for this condition was 5.3 degree aft (4.2 degree aft when corrected for tunnel wall effect). Absolute pressures were measured at $\mathrm{r} / \mathrm{R}=0.75,0.87$, and 0.97. The HART-I data were averaged over 64 revolutions.

Figure 2 shows the blade planforms for the five rotors, along with the location of the airfoils used and the pressure measurements. Figure 3 shows the blade twist distributions. Most of the rotor blades have about -8 degree of twist while the UH-60A has about -16 degree twist and its distribution is non-linear near the tip.

\section{CAMRAD II Modeling}

The H-34 in flight and wind tunnel, research Puma and UH-60A Black Hawk in flight, and model-scale BO-105 in wind tunnel were modeled in CAMRAD II as isolated rotors. The trim solution for the H-34 in flight and wind tunnel and the research Puma in flight solved for the controls that produced rotor thrust and first harmonic flapping to match the measured values, with the rotor shaft angle of attack fixed at the measured values. The trim solution for the UH-60A in flight and BO-105 in wind tunnel solved for the controls that produced rotor thrust and shaft pitch and roll moments to match the measured values, with the rotor shaft angle of attack fixed at the measured values.

The SA 349/2 in flight was modeled as a complete aircraft, with the Fenestron tail rotor replaced by an auxiliary anti-torque force. The trim solution for the SA 349/2 in flight solved for the controls and aircraft attitudes that produced zero total force and moment on the aircraft, with zero sideslip angle.

\section{Aerodynamic and Wake Model}

The CAMRAD II aerodynamic model for the rotor blade is based on lifting-line theory, using steady twodimensional airfoil characteristics and a vortex wake model. The rotor blade modeling problem of liftingline theory is unsteady, compressible, viscous flow about an infinite aspect-ratio wing, in a uniform flow consisting of the yawed free stream and the wake-induced velocity. This problem is modeled as two-dimensional, steady, compressible, viscous flow (airfoil tables), plus corrections. The corrections in particular account for 
swept and yawed flow, spanwise drag, and attached flow unsteady loads. Other corrections available, such as for static stall delay and dynamic stall, were not important for the operating conditions considered here.

The wake problem of lifting-line theory is an incompressible vortex wake behind the lifting-line, with distorted geometry and rollup. The lifting-line (bound vortex) is at the quarter chord (an approximation for the quadrupole line of second-order loading), and the three components of wake-induced velocity are evaluated at collocation points at the three-quarter chord (an approximation for the linearly varying induced velocity introduced by second-order wake). This model is generally second-order accurate for section lift, which significantly improves the calculation of bladevortex interaction loading, but less accurate for section moments.

The wake analysis calculates the rotor nonuniform induced-velocity using free wake geometry. The concentrated tip vortices are the key features of the rotor wake, important for performance, airloads, structural loads, vibration, and noise calculations. The formation of the tip vortices is modeled in CAMRAD II, not calculated from first principles. Two cases are examined here: a rolled-up model and a multiple-trailer with consolidation model. Because of its simplicity and efficiency, the rolled-up model has long been used for helicopter rotors. The multiple-trailer model has also been available, and with the consolidation feature has been applied recently with success to tiltrotor airloads calculations [9].

The rolled-up wake model is based on the assumption that a tip vortex forms at the outboard blade tip. The bound circulation can have the same sign all along the blade span, or there may be two bound circulation peaks (inboard and outboard peaks of opposite sign). These are the single-peak and dual-peak cases. The rolled-up wake is constructed from the magnitude and position of the peak bound circulation. For the single peak case, the maximum bound circulation is $\Gamma_{\max }$. In the far wake (where the rollup process is complete) there is a tip vortex with strength equal to $\Gamma_{\max }$ at the time that the wake element was created; and there is corresponding negative trailed vorticity with total strength $-\Gamma_{\max }$ in an inboard sheet. The tip vortex is modeled as a line segment with this strength and a small core radius. Any error in the assumed strength (because the vortex is partially or over rolled-up) is compensated for by the value used for the core radius. The detailed structure of the inboard vortex sheet is not calculated, so a single sheet element with trailed and shed vorticity is used. For the dual-peak case, the bound circulation can be positive over part of the blade span, but negative at the blade tip. In this case the tip vortex consists of the trailed vorticity from the outboard peak to the tip, hence has strength equal to the outboard peak bound circulation. The inboard wake sheet now has both positive and negative trailed vorticity, divided at the radial station corresponding to the inboard circulation peak. The trailed vorticity between the two circulation peaks might also roll up. For the current study, the single-peak model was used for the low speed conditions, and the dual-peak model was used for the high speed conditions.

The multiple-trailer model has the far wake trailed vorticity divided into several spanwise panels, to provide more detailed structure for the inboard vorticity, with consolidation of trailed lines in the wake geometry to model the rollup process. This model has a discrete trailed vortex line emanating from each of the aerodynamic panel edges. The calculation of the free wake geometry includes the distortion of all of these trailed lines. With multiple far wake trailed vorticity panels, the trailed lines at the aerodynamic panel edges can be consolidated into rolled-up lines, using the trailed vorticity moment to scale the rate of roll-up. The trailed vorticity is partitioned into sets of adjacent lines that have the same sign (bound circulation increasing or decreasing). For each set, the total strength $G$, centroid $r_{C}$, and moment (radius of gyration) $r_{G}$ of the trailed vorticity set are calculated:

$$
\begin{aligned}
G & =\int(-\partial \Gamma / \partial r) d r \\
G r_{C} & =\int(-\partial \Gamma / \partial r) r d r \\
G r_{G}^{2} & =\int(-\partial \Gamma / \partial r)\left(r-r_{C}\right)^{2} d r
\end{aligned}
$$

where $\Gamma$ is the bound circulation. It is assumed that all the vorticity in a set eventually rolls up into a single vortex, located at the centroid of the original vorticity distribution. The characteristic time $\left(r_{G}^{2} / \Gamma\right)$ is taken as a measure of the rate of consolidation

The wake models in CAMRAD II allow the vortex core radius to be defined in several ways. The vortex core radius is specified by a constant term (input fraction of chord, $r_{c 0} / c$ ); a term growing with wake age $\tau$ (using an input exponent $\mathrm{n}$, and $\tau_{1}=$ wake age for core radius of $100 \%$ chord); and a term that scales with the trailed vorticity moment. Hence, the general expression for the core radius is:

$$
r_{c}=c\left(r_{c 0} / c\right)+c\left(\tau / \tau_{1}\right)^{n}+f_{M} r_{G}
$$


where $r_{G}$ is the second moment about the centroid (the radius of gyration) of the trailed vorticity, evaluated at the time the vortex element was created. This moment is obtained by integrating the vorticity for all adjacent trailed lines of the same sign. Equating the moment of the trailed vorticity created behind the blade to the moment of the rolled-up vortex implies that the constant $f_{M}$ should be on the order of 1.0 (depending on the vorticity distribution in the vortex core). An additional feature of the core radius model in CAMRAD II is an option to suppress blade-vortex interaction at inboard radial stations.

\section{Results and Discussion}

The calculated blade section normal forces and pitching moments were compared with the flight and wind tunnel measurements. The calculations have been conducted with the identical analysis options for all rotors. The azimuthal step size was 15 degree for the aerodynamic and structural-dynamic calculations. A constant vortex core radius of $20 \%$ chord $(0.2 \mathrm{c})$ was used for the rolled-up wake model. The consolidation model used a compression form, with linear dependence of the rollup fraction as wake age. The vortex core radius had a constant value of $50 \%$ mean chord. For all cases, an elastic blade model was used to calculate the airloads.

\section{Airloads Correlation at Low Speed}

Figures 4 through 8 show non-dimensionalized blade section normal force of the $\mathrm{H}-34$, research Puma, SA 349/2, UH-60A Black Hawk, and BO-105 rotors at an advance ratio of $0.129,0.141,0.14,0.149$, and 0.15 respectively. Correlation is shown at three radial locations for each aircraft. At low speed in the transition regime, the airloads are mainly determined by the interaction between the blades and the vortices trailed from the preceding blades [8]. All the flight test data show a sharp "down-up" impulse on the advancing blade and an opposite impulse on the retreating blade near the blade tip.

A free wake is important for the prediction of airloads in low speed flight. The analysis used two wake models: a rolled-up and a multiple-trailer wake with consolidation. The multiple-trailer wake with consolidation, which showed better airload correlation for the UH-60A [10] and BO-105 [11] rotors, was applied to five different rotors to assess the general utility of this wake model.

The analysis with the rolled-up wake model captured the rapid changes of airloads on both the advancing and retreating sides reasonably well for the $\mathrm{H}-34$, research Puma, and SA 349/2. The calculated airloads differed significantly from the measurements for the UH-60A and BO-105. The rolled-up wake model significantly overpredicted the magnitude of the "downup" impulse on the advancing side for the UH-60A and underpredicted at $\mathrm{r} / \mathrm{R}=0.75$ and 0.87 for the $\mathrm{BO}-105$.

Better correlation was obtained for the UH-60A and BO105 by using a free wake geometry calculation method that combines the multiple-trailer wake with a simulation of the tip vortex formation process (consolidation). However, the influence of the multiple-trailer with consolidation model was different for the two rotors. For the UH-60A, the multiple-trailer with consolidation model reduced blade-vortex interaction compared to the rolled-up wake on the advancing side, and thus improved the correlation. For the BO-105, the multiple-trailer with consolidation model increased blade-vortex interaction at $\mathrm{r} / \mathrm{R}=0.75$ and 0.87 on the advancing side, and thus improved the correlation. An overprediction was still observed on the front of the rotor disk at some of the radial locations. However, the correlation with the multiple-trailer wake, in general, was better than with the rolled-up wake on the front of the rotor disk. Particularly good correlation was observed at $\mathrm{r} / \mathrm{R}=0.965$ for the $\mathrm{UH}-60 \mathrm{~A}$ and $\mathrm{r} / \mathrm{R}=0.75$ for the BO-105. For the other rotors, the multiple-trailer with consolidation model had less influence on the accuracy of the prediction of the normal force. There was a small magnitude change on the advancing and retreating sides, but, in general, the phase was not changed.

It should be noted that the high frequency loading observed in the BO-105 test data is the source of BVI noise. A smaller time step would be required to capture the high frequency content. However, the scope of the present study is loads and vibration, thus the 15 degree time step was consistently used.

Figure 9 shows blade section pitching moment correlation. The measured pitching moment values for the BO-105 have not yet been published for the HART-I, thus the comparison with the analysis was not included in this paper. The measured pitching moment data show more high frequency content than the normal force data. In general, the correlation is poor with either of the wake models. Again, the difference between the two wake models is larger for the UH-60A.

\section{Airloads Correlation at High Speed}

Figures 10 through 13 show blade section normal force of the H-34, research Puma, SA 349/2, and UH-60A Black Hawk rotors at an advance ratio of $0.39,0.362,0.361$, and 0.368 respectively. At high speed, the interaction between the blade and wake diminishes as the rotor disk is tilted further forward and the tip vortices are convected 
more quickly away from the rotor disk. The airloads on the blade tip region are characterized by negative lift at the end of the first quadrant and the beginning of the second quadrant [8]. This negative loading occurs on the H-34, research Puma, and UH-60A Black Hawk rotors. The SA 349/2 flight test data do not show the negative loading, although the flight condition $\left(C_{T} / \sigma\right.$ and $\left.\mu\right)$ is similar with the other rotor tests.

Comparison of calculation using the comprehensive analysis CAMRAD II with the research Puma flight data (Fig. 11) shows, in general, good agreement with the measurements in both magnitude and phase. However, good agreement is not obtained for the other rotors examined. The test data show that the minimum normal force appears almost at the same azimuth angle along the radial locations examined for each rotor. The analysis also shows the same trends. Although the amplitude shows fair to good correlation for some of the rotors, there is about 20 to 45 degree phase difference at all blade stations examined. The multiple-trailer with consolidation model has a small influence on the prediction of normal force. The phase difference between the test data and analysis does not appear to come from the wake modeling.

Figure 14 shows blade section pitching moment correlation. The correlation for the H-34 is fair to good. In general, the correlation is poor for the other rotors examined.

\section{Vibratory Airloads at Low and High Speeds}

Figures 15 through 19 show the blade vibratory normal force at low speed. Steady, $1 / \mathrm{rev}$, and $2 / \mathrm{rev}$ harmonics have been removed from the test data and analyses for the H-34, research Puma, UH-60A, and BO-105. Steady and $1 /$ rev harmonics have been removed for the 3-bladed SA 349/2. The harmonics retained are the vibratory loads that are transmitted through the shaft to the fuselage and produce vibration of multiples of $N_{b}$ per rev. For all of these rotors the vibratory loading is dominated by a sharp "down-up" impulse on the advancing blade and an opposite impulse on the retreating blade and this loading is more dominant near the blade tip.

Both the rolled-up and multiple-trailer with consolidation wake models show fair to good correlation for the H-34, research Puma, and SA 349/2. The rolled-up wake model overpredicted the magnitude of the "down-up" impulse on the advancing side at all the radial locations examined for the research Puma and at $\mathrm{r} / \mathrm{R}=0.97$ for the SA 349/2. The multiple-trailer with consolidation model tended to reduce the magnitude of the "down-up" impulse on the advancing side, and thus, for some cases, resulted in underprediction.
A larger difference between the two wake models appeared for the UH-60A and BO-105. The multipletrailer with consolidation model showed better correlation than with the rolled-up wake, especially on the advancing side and the front of the rotor disk. The correlation with the multiple-trailer with consolidation model was better for the vibratory loading (3/rev and higher) than for steady to $2 /$ rev loading.

Figures 20 through 23 show the blade vibratory normal force at high speed. The higher harmonic airloads show a sharp up-down impulse in the 1st and 2nd quadrants. This mode of airload excitation was found to be present to a large extent in almost all the data examined. The correlation, in general, is poor to fair. For the H-34, there was a significant difference between the data and the analysis for both magnitude and phase. Better correlation was observed for the research Puma compared to the other rotors. Although there was an underprediction of peak-to-peak magnitude, reasonably good correlation was observed for phase. For the SA 349/2, the analysis shows poor correlation on both magnitude and phase. Unlike with the other rotors where the analysis underpredicted the magnitude, the analysis significantly overpredicted the magnitude, especially at $\mathrm{r} / \mathrm{R}=0.75$ and 0.88 , due to strong $2 / \mathrm{rev}$ harmonics. For the UH-60A, the calculation shows nearly an opposite phase on the advancing side.

\section{Comparison of Blade Oscillatory Normal Force}

Figures 24 and 25 compare the blade oscillatory normal force near $\mathrm{r} / \mathrm{R}=0.9$ where the steady airloads have been removed from the test data and calculations. The normal force values for the $\mathrm{H}-34$ are at $\mathrm{r} / \mathrm{R}=0.9$, for the research Puma and UH-60A at $\mathrm{r} / \mathrm{R}=0.92$, for the SA 349/2 at $\mathrm{r} / \mathrm{R}=0.88$, and for the $\mathrm{BO}-105$ at $\mathrm{r} / \mathrm{R}=0.87$. In these figures the test data and the calculated loads are grouped separately.

Figure 24 shows the blade oscillatory normal force at low speed. Steady, level flight test data $(\mathrm{H}-34$, research Puma, SA 349/2, and UH-60A) show that the minimum peak on the advancing side appears at 72 to 82 degree azimuth angle and the minimum peak on the retreating side appears at 285 to 300 degree azimuth angle. For the BO-105 in the descending condition, the minimum peaks appear around 45 and 310 degree azimuth angle. The normal force values on the front of the rotor disk are close to zero for the H-34, research Puma, and SA 349/2. However, strong negative values are observed for the UH$60 \mathrm{~A}$ and $\mathrm{BO}-105$ rotors.

The analysis with the rolled-up wake shows similar phase for the H-34, research Puma, and SA 349/2: the minimum peak on the advancing side at 75 degree and on 
the retreating side at 285 to $300 \mathrm{deg}$. The phase difference is observed for the UH-60A compared to the test data and the analysis of the other rotors. The minimum peak on the advancing side is at $60 \mathrm{deg}$ for the UH-60A. The normal force values on the front of the rotor disk are close to zero for all the rotors. The multiple-trailer wake captures strong negative values on the front of the rotor disk observed for the UH-60A and BO-105 rotors.

Figure 25 shows the blade oscillatory normal force at high speed. There are many similarities in the measured normal force among the H-34, research Puma, and SA 349/2 rotors. The UH-60A data show a stronger negative peak probably because the UH-60A blade has more twist than the other rotor blades. The negative peak appears between 108 degree for the research Puma and 135 degree for the UH-60A. However, the analysis shows that the minimum normal force appears at the 90 degree azimuth angle regardless of the rotor except for the research Puma, where the minimum normal force appears at $105 \mathrm{deg}$.

\section{Quantitative Airloads Correlation}

To characterize the accuracy of the correlation, the normal force data have been examined quantitatively. Figure 26 shows the harmonic correlation of the blade normal force at $\mathrm{r} / \mathrm{R}=0.865$ for the UH-60A at $\mu=0.149$. The steady, sine, and cosine harmonic coefficients of the calculation were plotted against the harmonic coefficients of the measurement up to 10/rev with the rolled-up and multiple-trailer wake models. The 45 degree diagonal line represents a perfect match between analysis and test. The calculated normal force coefficients lie above the 45 degree line if the analysis overpredicts, and below the line if the analysis underpredicts. The correlation is assessed by fitting a least squares regression line and computing the slope, m. A second measure is the correlation coefficient, $r$, which provides an indication of dispersion. A third measure is the RMS error from the 45 degree line.

Figures 27 and 28 show the m, r, and RMS error values along the blade span for the H-34 and UH$60 \mathrm{~A}$ respectively. These two data sets contain airloads data at inboard sections of the blade. Steady to $10 / \mathrm{rev}$ harmonic coefficients are used for the correlation. Near the blade tip, the multiple-trailer with consolidation wake model shows similar or slightly better correlation for the $\mathrm{H}-34$ and significantly better correlation for the UH$60 \mathrm{~A}$. However, at inboard and mid-span, the multipletrailer with consolidation wake model, in general, shows somewhat worse correlation.

For the correlation of 3 to $10 /$ rev harmonics, as shown in Figs. 29 and 30, the multiple-trailer with consolidation wake model shows worse correlation at inboard and midspan for the H-34.

\section{Parametric Study of High Speed Airload Calculation}

The calculation shows good phase correlation at high speed for the research Puma while the same analysis cannot predict the correct phase for the other rotors examined. It is important to understand why the same analysis shows good correlation for one rotor but not the others. This can be examined using the research Puma and UH-60A rotors because the two articulated rotors have a similar size and both have swept tips.

There are many differences between the two rotors, structurally and aerodynamically. The research Puma has the NACA 0012 airfoil along the span while the UH60A blade uses two airfoils, the SC1095 and SC1094 R8. Another significant difference between the two rotors is the blade twist, as shown in Fig. 3. The Puma has about -8 degree of twist while the UH-60A has about -16 degree twist and its distribution is non-linear near the tip. Figure 31 shows that the two blades have almost same chord length at the straight portion of a blade, but the chord length is increased for the Puma near $r / R=0.9$. The quarter chord of the Puma is forward of the quarter chord of the UH-60A near $\mathrm{r} / \mathrm{R}=0.9$ and then moves aft of the quarter chord of the UH-60A near the tip. There is also a slight difference in flight and trim condition.

The parameters investigated in this study are: airfoil, twist, structural tip design (elastic axis and center of gravity location), aerodynamic tip design (chord length and quarter chord location), and trim (flight) condition. The effects of those parameters on the prediction of blade airloads were examined by replacing the UH-60A quantities with the equivalent research Puma quantities. For example, the SC1095 and SC1094 R8 airfoils were replaced with the NACA 0012 airfoil to understand the influence of airfoil on the prediction of blade airloads.

Figure 32 shows the blade normal force and pitching moment of the UH-60A at $r / R=0.92$. The structural tip design and trim condition have a small influence on the normal force prediction. The airfoil and twist change the magnitude, but not the phase. The only parameter that changed the phase of the normal force is the aerodynamic tip design (chord length and quarter chord location). The negative peak appears at 105 degree azimuth angle by using the chord length and quarter chord of the Puma. However, the quarter chord or chord length alone did not change the phase.

Most of the parameters have a small influence on the pitching moment prediction. The airfoil change from the cambered to symmetric produces less negative pitching moment. The aerodynamic tip design shows better pitching moment correlation. This additional pitching moment appears to shift the normal force phase by 15 deg. 


\section{Conclusions}

Blade section normal force and pitching moment were investigated for various rotors operating at transition and high speeds: H-34 in flight and wind tunnel, SA 330 (research Puma), SA 349/2, UH-60A full-scale and BO-105 model (HART-I). The H-34, research Puma, SA 349/2, UH-60A data represent steady, level flight conditions and the BO-105 data represent descending flight condition. Measured data from flight and wind tunnel tests were compared with calculations obtained using the comprehensive analysis CAMRAD II.

From this study the following conclusions were obtained:

\section{Low Speed}

1. All the flight test data show a sharp "down-up" impulse on the advancing blade and an opposite impulse on the retreating blade.

2. The analysis with a rolled-up wake model captures the rapid changes of airloads on both the advancing and retreating sides reasonably well for the $\mathrm{H}-34$, research Puma, and SA 349/2. The calculated airloads differ significantly from the measurements for the UH-60A and BO-105.

3. Better correlation is obtained for the UH-60A and BO-105 by using a free wake geometry calculation method that combines the multiple-trailer wake with a simulation of the tip vortex formation process (consolidation).

4. The significant improvement in the normal force correlation with the multiple-trailer wake model appears to happen near the blade tip. In general, worse correlation is observed than with the rolledup wake model at inboard and mid-span for the H-34 and UH-60A, especially for the vibratory components.

5. The measured pitching moment data show more high frequency content than the normal force data. In general, the correlation is poor with either of the wake models.

\section{High Speed}

1. In high speed flight, the airloads on the blade tip region are characterized by negative lift at the end of the first quadrant and the beginning of the second quadrant. This negative loading occurs on the H-34, research Puma, and UH-60A Black Hawk rotors. The SA 349/2 flight test data do not show the negative loading, although the flight condition $\left(C_{T} / \sigma\right.$ and $\left.\mu\right)$ is similar with the other rotor tests.

2. The comparison of the calculations with the research Puma flight data shows, in general, good agreement with the measurements in both magnitude and phase. However, poor agreement is obtained for the other rotors examined. There is about 20 to 45 degree phase difference at all blade stations examined.

3. The correlation of the pitching moment for the $\mathrm{H}-$ 34 is fair to good. In general, the correlation is poor for the other rotors examined.

4. The parametric study shows that the Puma tip design (quarter chord location and chord length) has an important influence on the phase correlation.

\section{Appendix}

In the present study, flight and wind tunnel test cases from various rotors [1-6] have been carefully selected so that they represent similar test conditions at transition and high speeds. Figure A.1 shows the thrust and advance ratio of all the available test data. Only steady, level flight conditions were included. The test data cover a wide range of thrust and advance ratio. These data sets provide a valuable resource that can be used for future research.

Table A.1 shows the spanwise location of the pressure transducers and the number of transducers used at each spanwise locations. Earlier tests such as H-34 in flight and wind tunnel used differential pressure transducers and later tests used absolute pressure transducers. The number of transducers is an important factor for the accurate integration of airloads, especially pitching moment.

\section{References}

[1] Scheiman, J., "A Tabulation of Helicopter RotorBlade Differential Pressures, Stresses, and Motions as Measured in Flight," NASA TM X-952, 1964.

[2] Rabbott, J. P. Jr., Lizak, A. A., and Paglino, V. M., "A Presentation of Measured and Calculated FullScale Rotor Blade Aerodynamic and Structural Loads," USAAVLABS TR 66-31, 1966.

[3] Bousman, W. G., Young, C., Gilbert, N. E., Strawn, R. C., Miller, J. V., Maier, T. H., Costes, M., and Beaumier, P., "A Comparison of Lifting-Line and CFD Methods with Flight Test Data from a Research Puma Helicopter," NASA TM 110421, October 1996. 
[4] Heffernan, R. M., and Gaubert, M., "Structural and Aerodynamic Loads and Performance Measurements of an SA349/2 Helicopter with an Advanced Geometry Rotor," NASA TM 88370, November 1986.

[5] Kufeld, R. M., Balough, D. L., Cross, J. L., Studebaker, K. F., Jennison, C. D., and Bousman, W. G., "Flight Testing of the UH-60A Airloads Aircraft," American Helicopter Society 50th Annual Forum Proceedings, Washington D.C., May 1994.

[6] Splettstoesser, W. R., Heller, H., Mercker, E., Preisser, J. S., and Yu, Y. H., "The HART Programme, a Quadrilateral Cooperative Research Effort," American Helicopter Society 51st Annual Forum Proceedings, Ft. Worth, Texas, May 1995.

[7] Johnson, W., "Technology Drivers in the Development of CAMRAD II," American Helicopter Society Aeromechanics Specialist Meeting, San Francisco, California, January 1994.

[8] Hooper, W. E., "The Vibratory Airloads of Helicopter Rotors," Vertica, Vol 8, No. 2, 1984.

[9] Johnson, W., "Influence of Wake Models on Calculated Tiltrotor Aerodynamics," American Helicopter Society Aerodynamics, Acoustics, and Test and Evaluation Technical Specialist Meeting Proceedings, San Francisco, CA, January 2002.

[10] Yeo, H., and Shinoda, P. M., "Investigation of Rotor Loads and Vibration at Transition Speed," American Helicopter Society 58th Annual Forum Proceedings, Montreal, Canada, June 2002.

[11] Lim, J. W., and Yu, Y. H., "Prediction of BladeVortex Induced Airloads with a Multiple-Trailer Free Wake Model," AHS International Meeting on Advanced Rotorcraft Technology and Life Saving Activities Proceedings, Tochigi, Japan, November 2002. 
Table 1: Rotor Parameters and Operating Conditions

\begin{tabular}{lcccccc}
\hline \hline & H-34 & H-34 & SA 330 & SA 349/2 & UH-60A & BO-105 \\
\hline Configuration & articulated & articulated & articulated & articulated & articulated & hingeless \\
$N_{b}$ & 4 & 4 & 4 & 3 & 4 & 4 \\
$\sigma$ & 0.0622 & 0.0622 & 0.091 & 0.0627 & 0.0826 & 0.077 \\
Test & flight & wind tunnel & flight & flight & flight & wind tunnel \\
Radius (in) & 336.0 & 336.0 & 296.7 & 206.7 & 322.0 & 78.7 \\
Scale & full-scale & full-scale & full-scale & full-scale & full-scale & model-scale \\
& & & & & & \\
Low speed & & & & & & \\
$C_{T} / \sigma$ & 0.075 & & 0.070 & 0.065 & 0.079 & 0.057 \\
$\mu$ & 0.129 & & 0.141 & 0.140 & 0.149 & 0.150 \\
$\alpha_{T P P}$ (deg) & 4.1 & & 1.3 & 1.2 & 0.8 & -4.2 \\
$M_{90, \text { tip }}$ & 0.628 & & 0.672 & 0.719 & 0.740 & 0.735 \\
High speed & & & & & & \\
$C_{T} / \sigma$ & & 0.060 & 0.070 & 0.071 & 0.079 & \\
$\mu$ & & 0.390 & 0.362 & 0.361 & 0.368 & \\
$\alpha_{T P P}$ (deg) & & & 7.5 & 7.5 & 8.0 & \\
$M_{90, \text { tip }}$ & & 0.803 & 0.803 & 0.872 & 0.878 & \\
\hline \hline
\end{tabular}

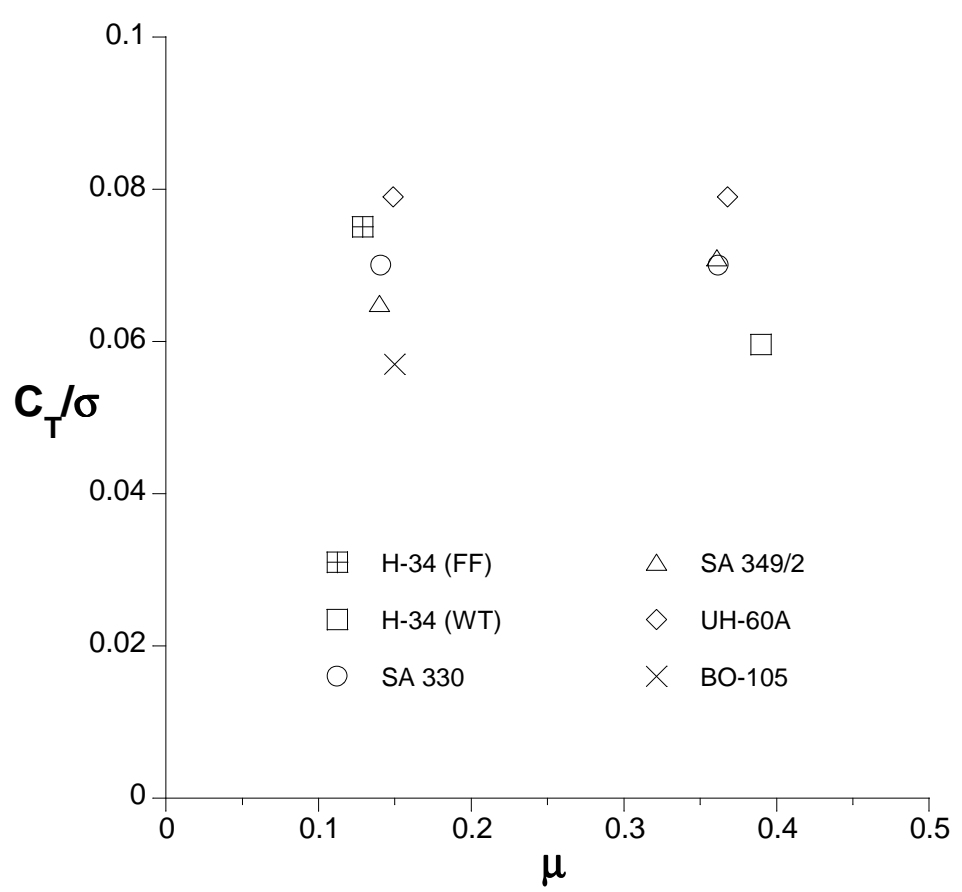

Fig. 1: Rotor thrust coefficient and advance ratio of test data examined 
$\mathrm{H}-34$

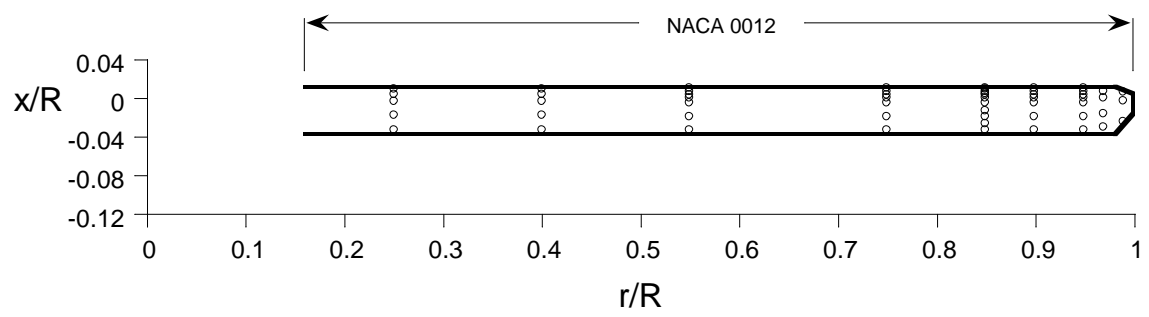

SA 330 (research Puma)

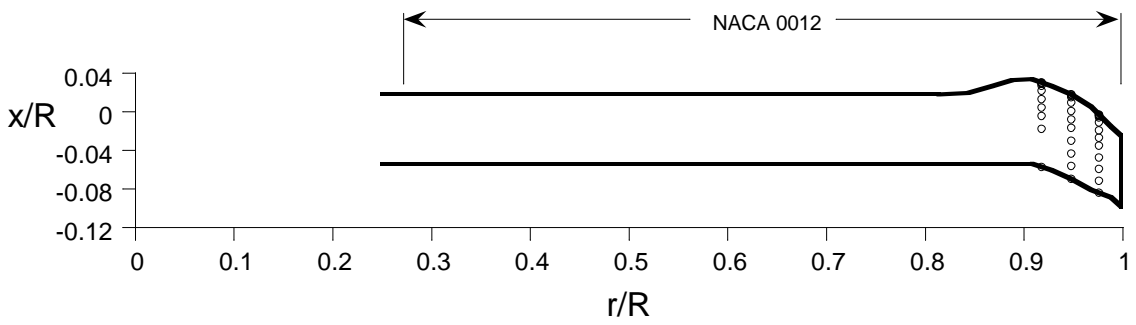

SA 349/2

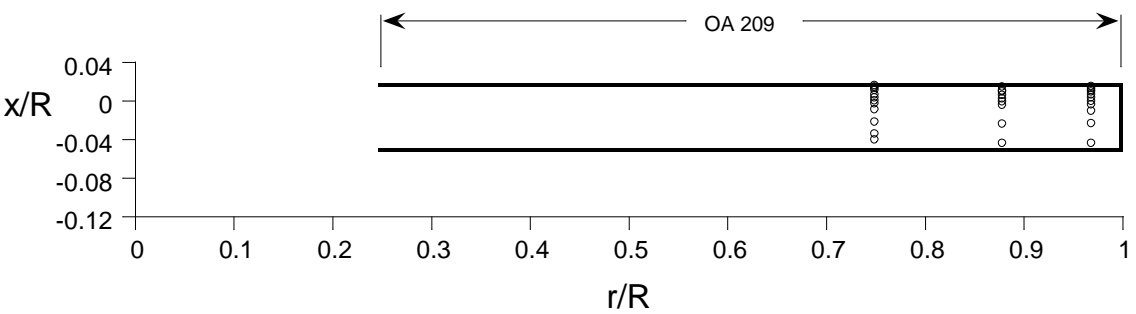

UH-60A

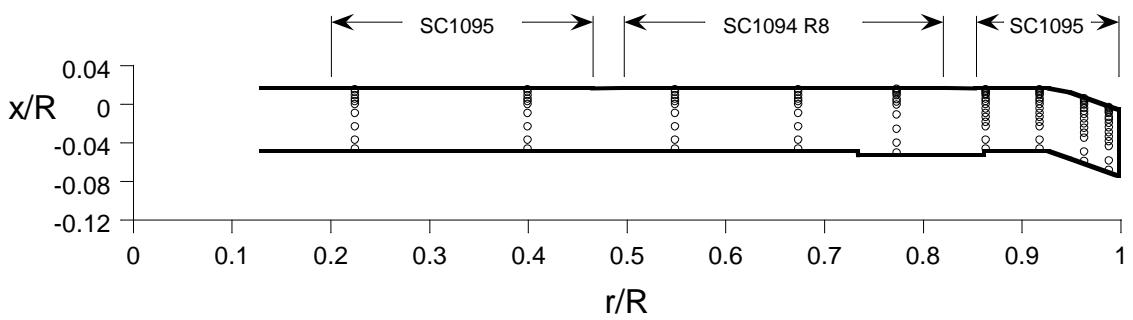

Model-scale BO-105

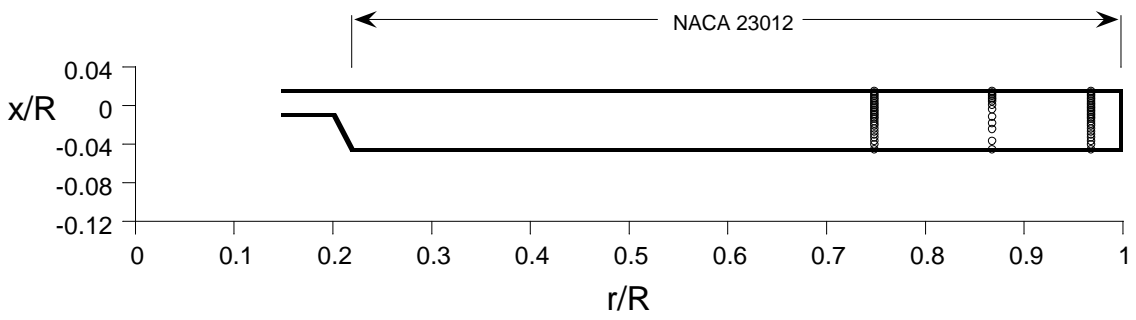

Fig. 2: Blade planforms 


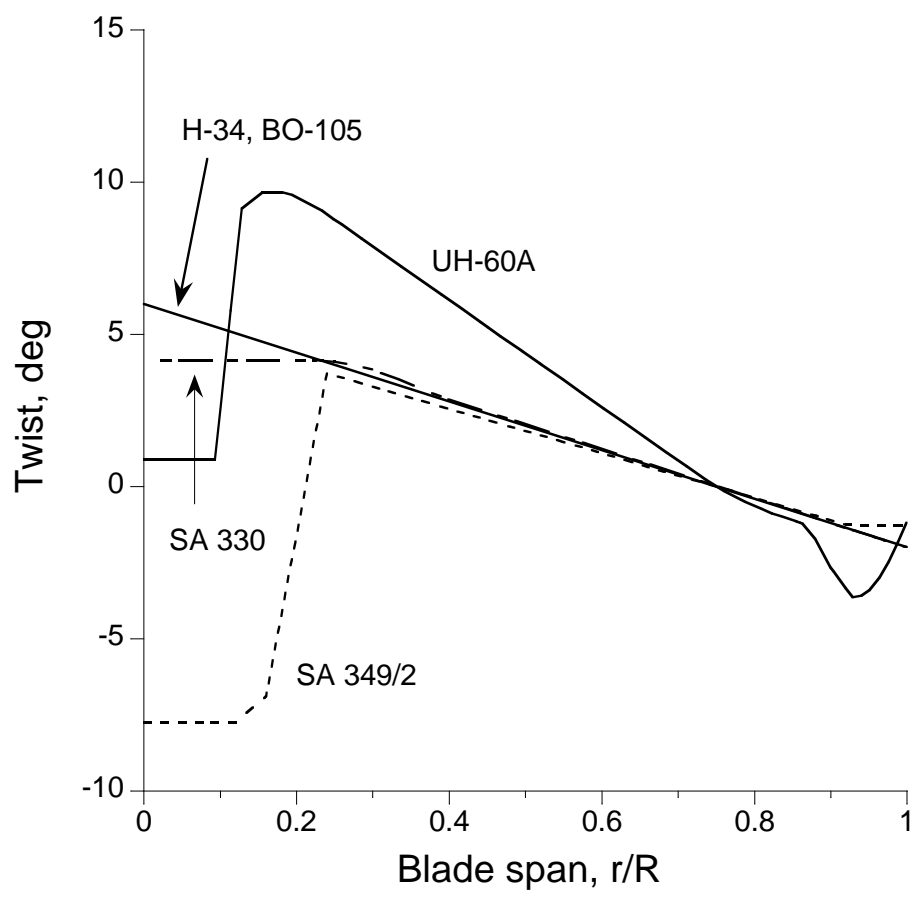

Fig. 3: Blade twist angle 


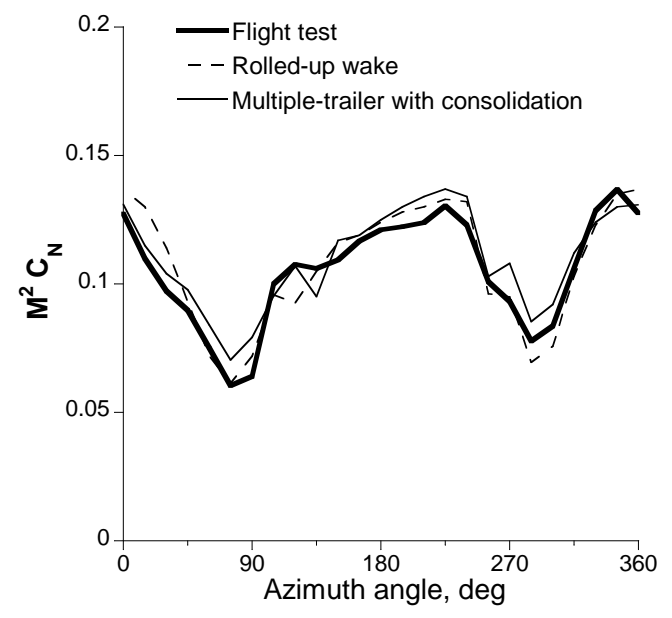

(a) $r / R=0.85$

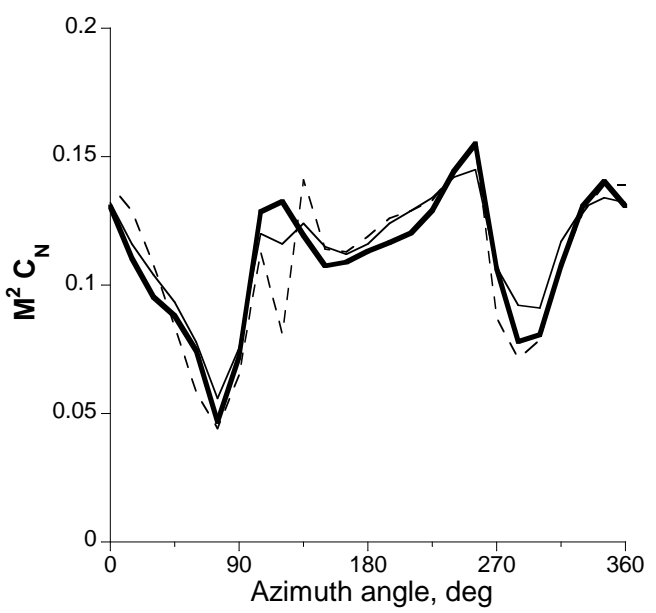

(b) $r / R=0.90$

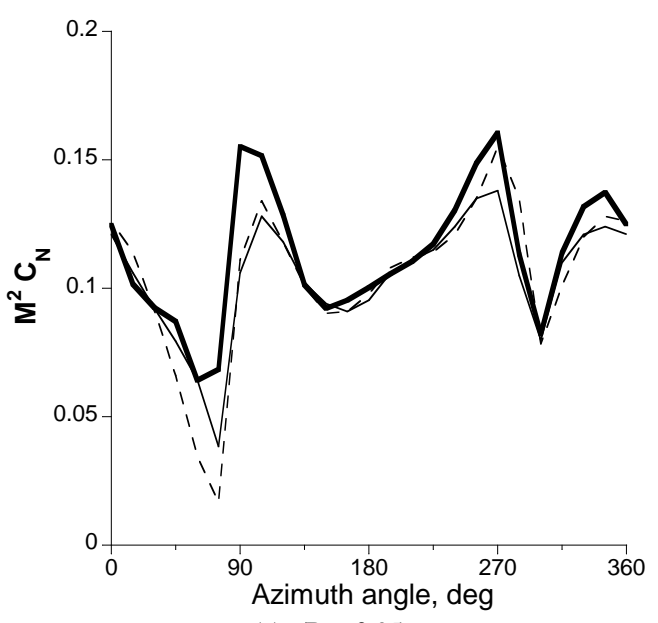

(c) $r / R=0.95$

Fig. 4: Calculated and measured blade normal force for $\mathrm{H}-34$ at $\mu=0.129$

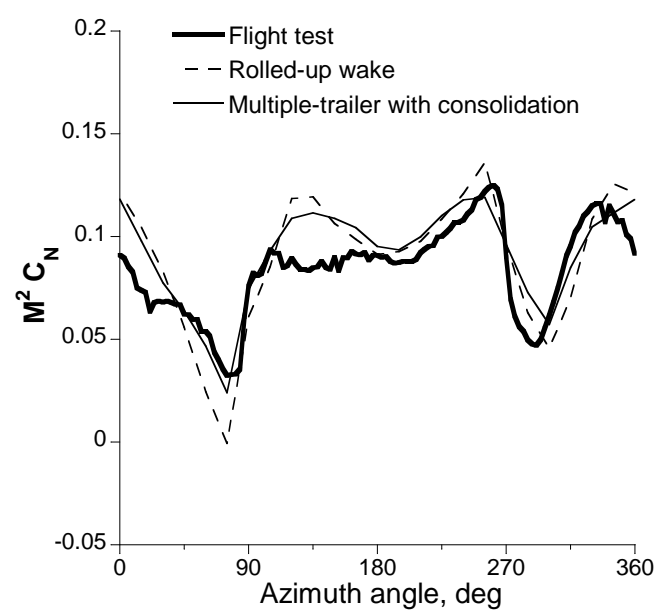

(a) $r / R=0.92$

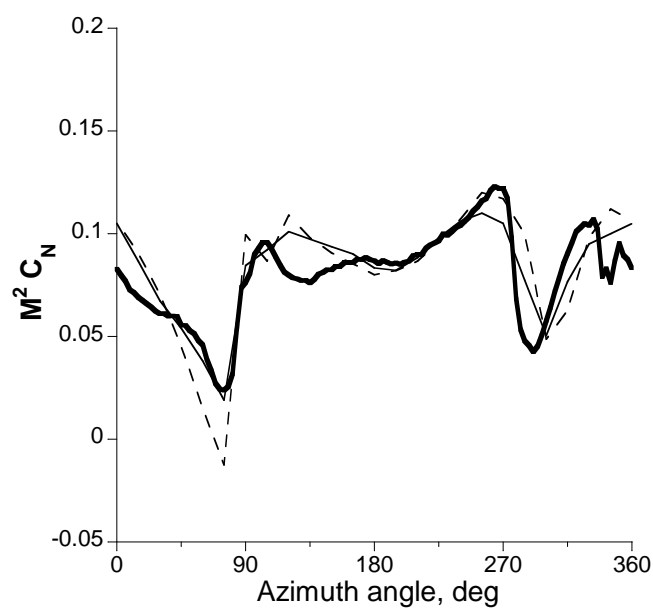

(b) $r / R=0.95$

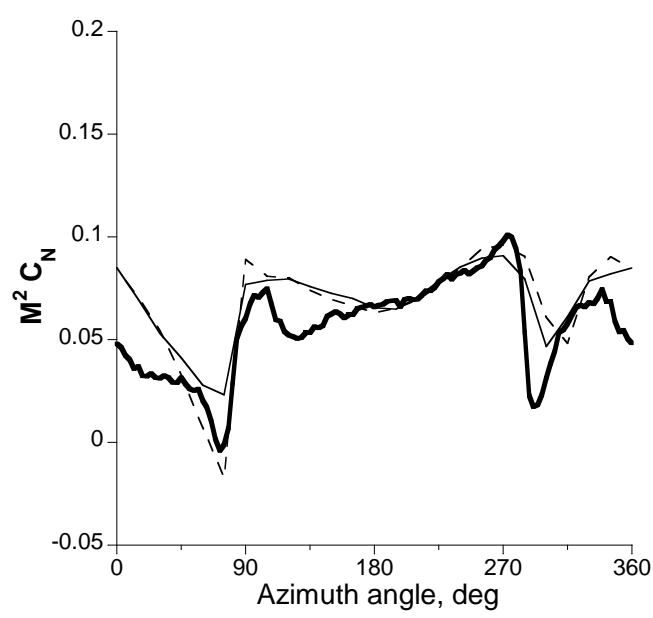

(c) $r / R=0.978$

Fig. 5: Calculated and measured blade normal force for SA 330 at $\mu=0.141$ 


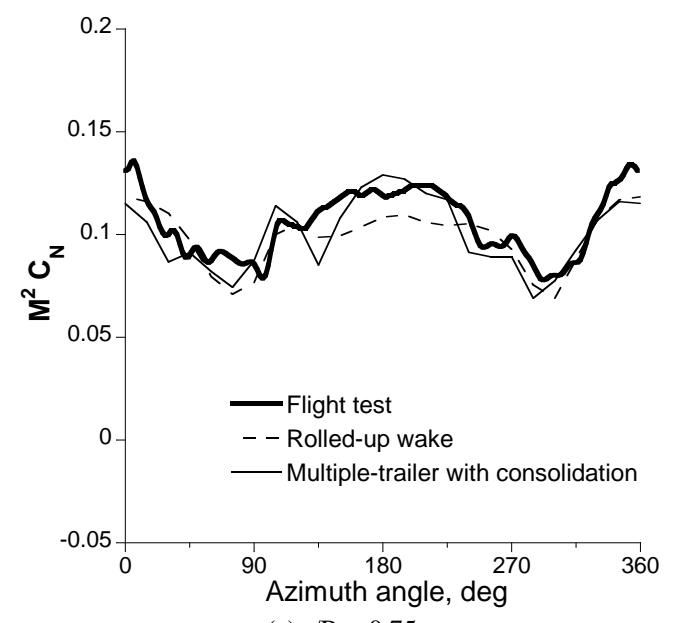

(a) $\mathrm{r} / \mathrm{R}=0.75$

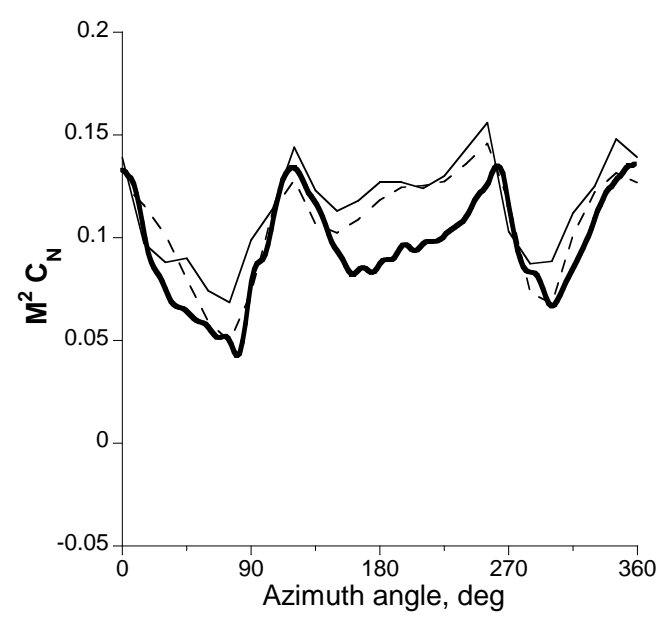

(b) $r / R=0.88$

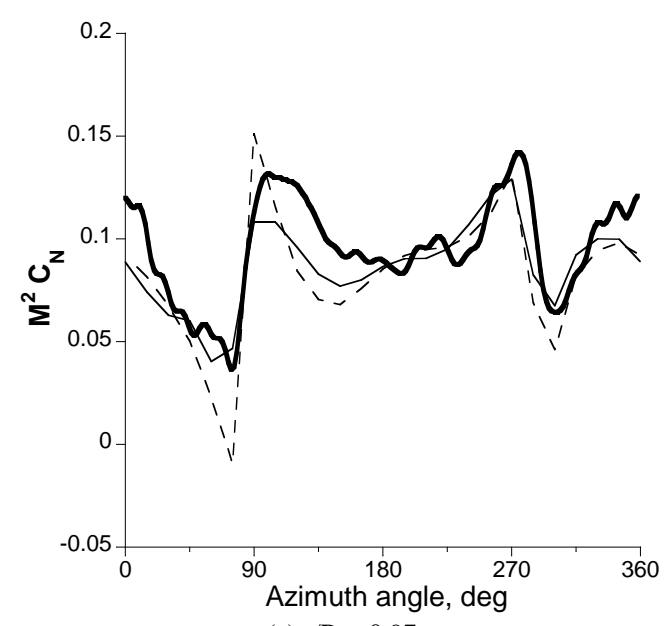

(c) $r / R=0.97$

Fig. 6: Calculated and measured blade normal force for SA $349 / 2$ at $\mu=0.14$

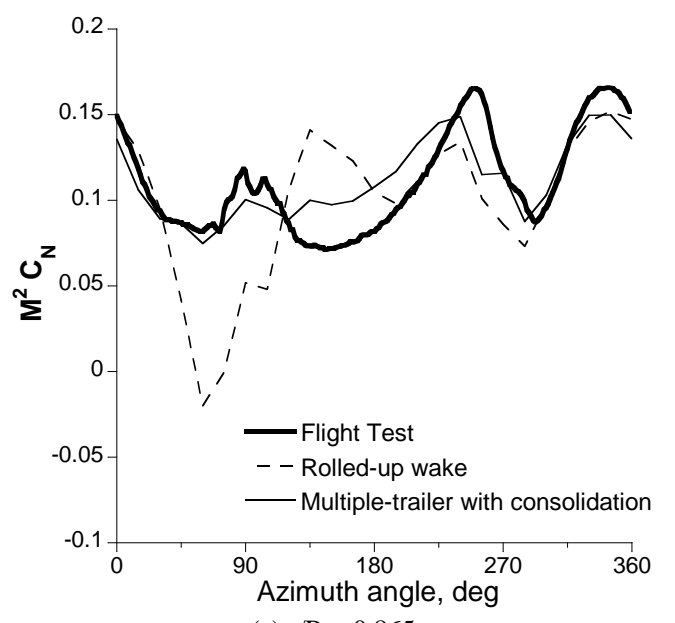

(a) $\mathrm{r} / \mathrm{R}=0.865$

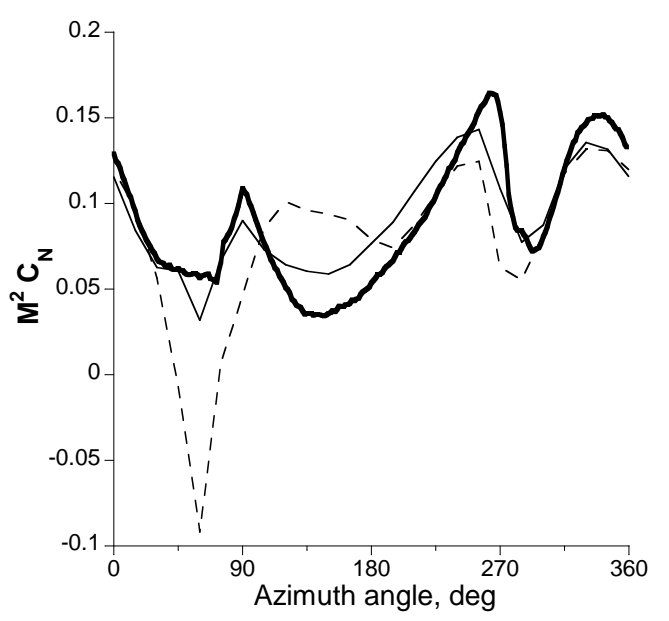

(b) $r / R=0.92$

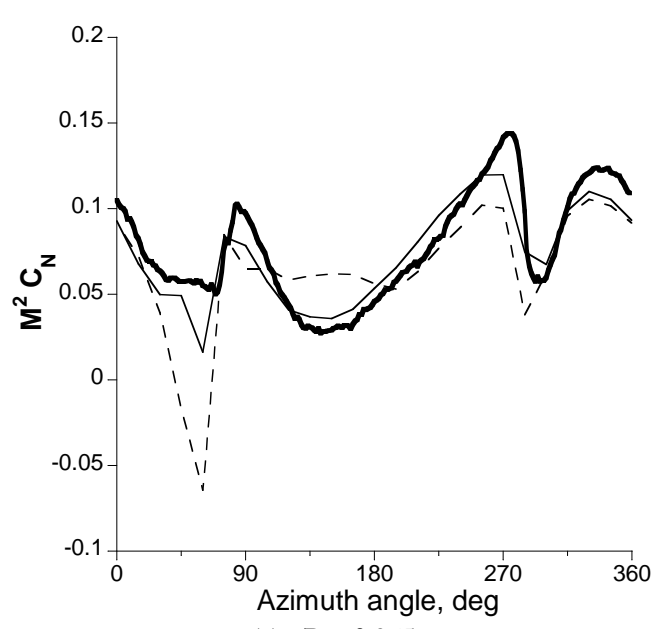

(c) $r / R=0.965$

Fig. 7: Calculated and measured blade normal force for UH-60A at $\mu=0.149$ 

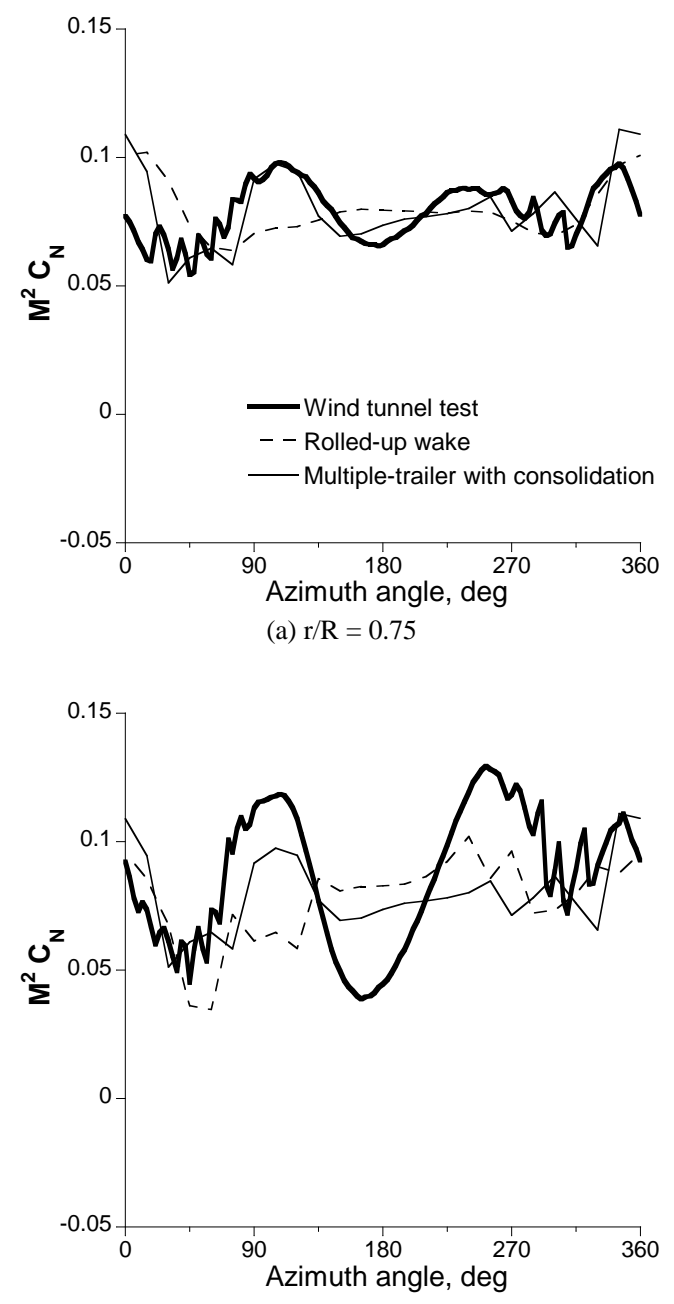

(b) $r / R=0.87$

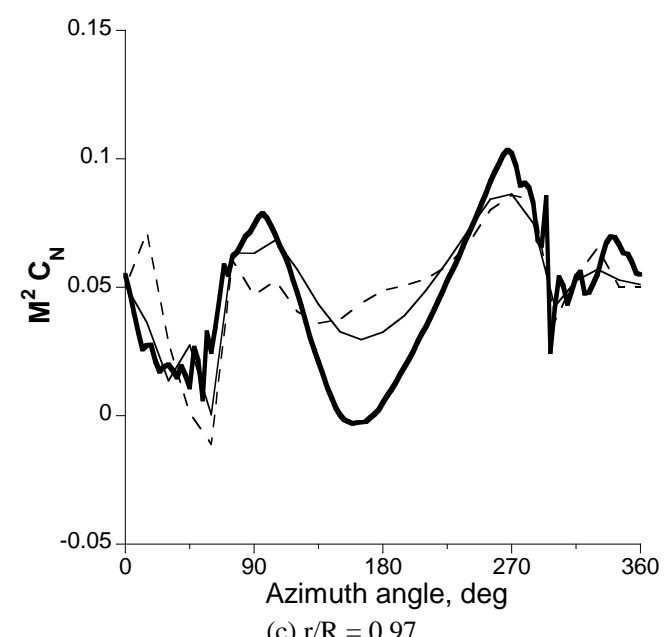

Fig. 8: Calculated and measured blade normal force for BO- 105 at $\mu=0.15$ 


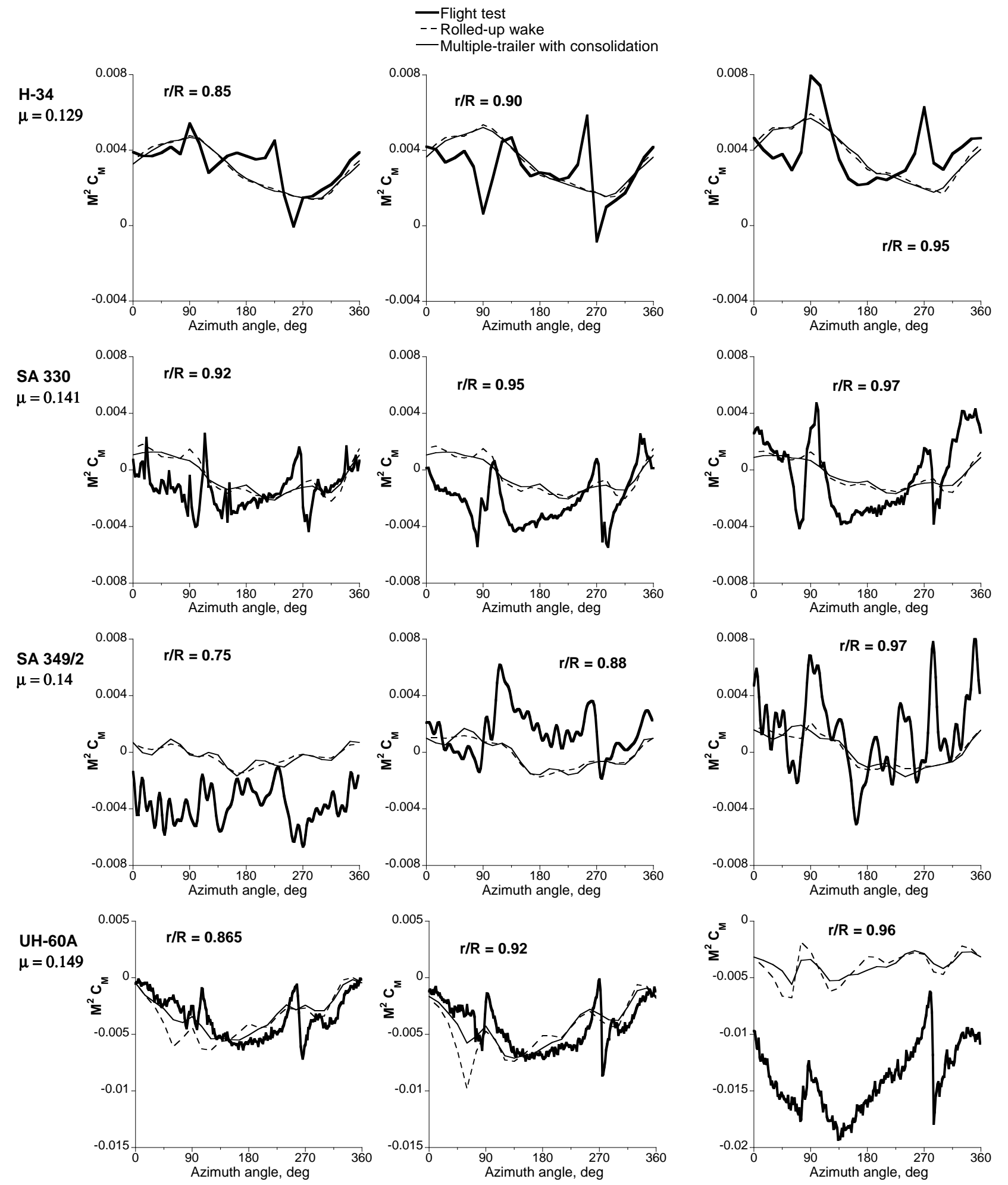

Fig. 9: Calculated and measured blade pitching moment at low speed 


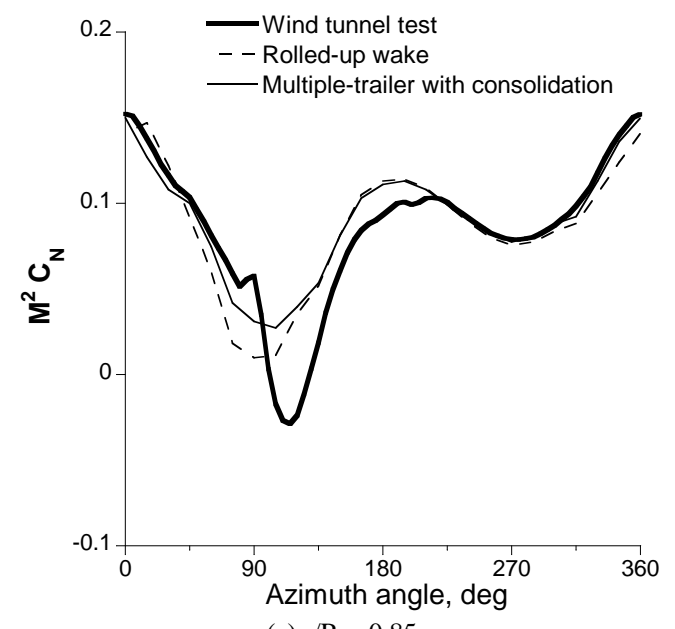

(a) $\mathrm{r} / \mathrm{R}=0.85$

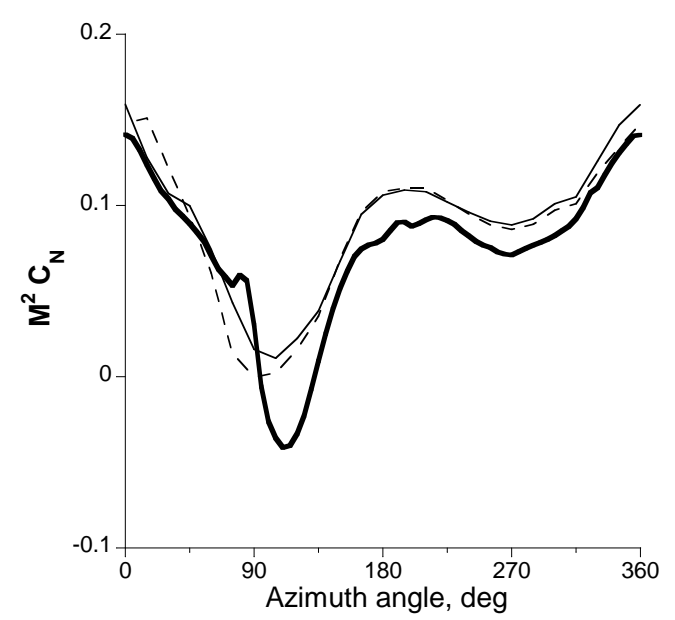

(b) $r / R=0.90$

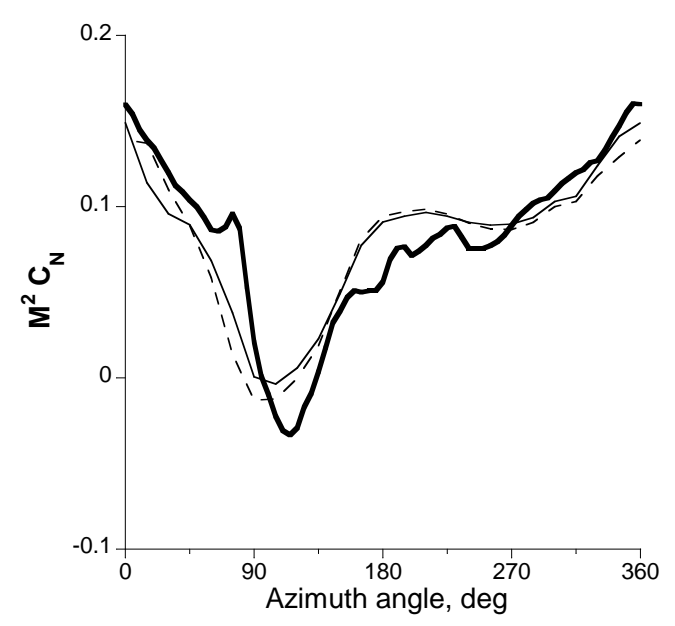

(c) $\mathrm{r} / \mathrm{R}=0.95$

Fig. 10: Calculated and measured blade normal force for H-34 at $\mu=0.39$

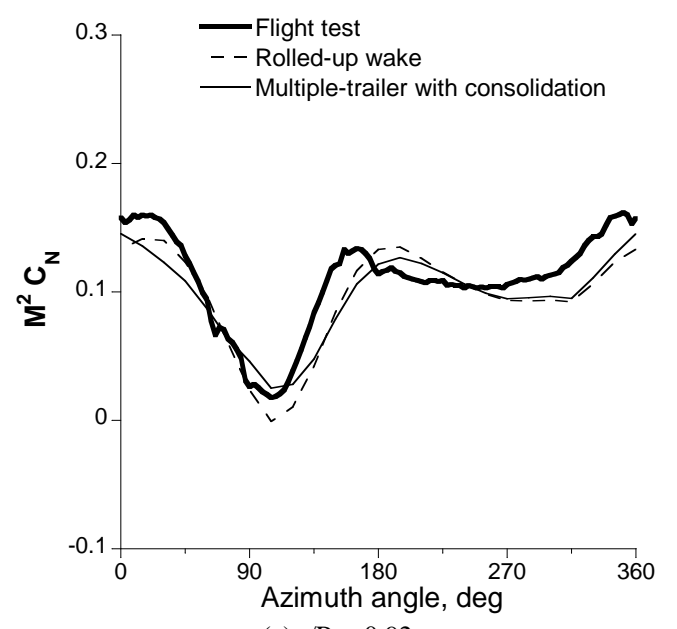

(a) $\mathrm{r} / \mathrm{R}=0.92$

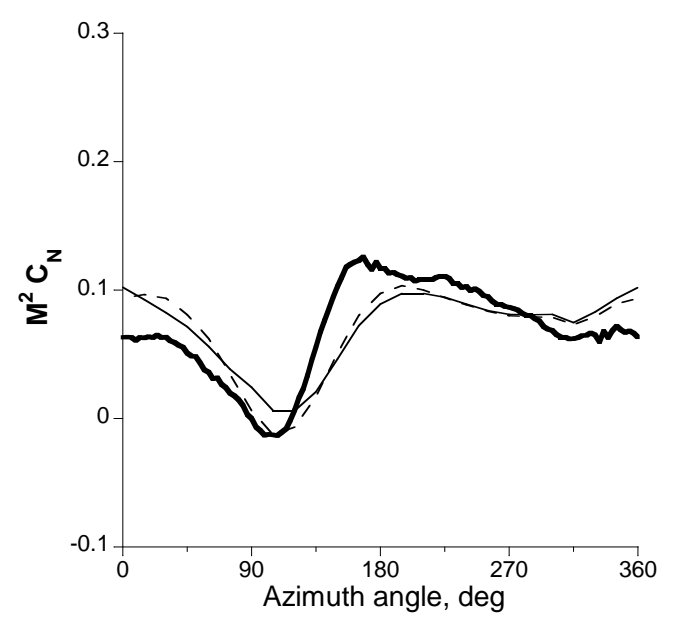

(b) $r / R=0.95$

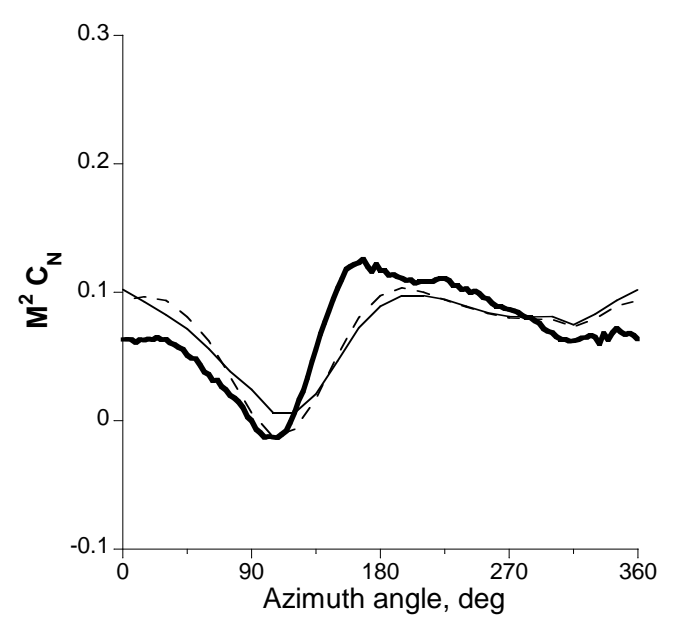

(c) $r / R=0.978$

Fig. 11: Calculated and measured blade normal force for SA 330 at $\mu=0.362$ 


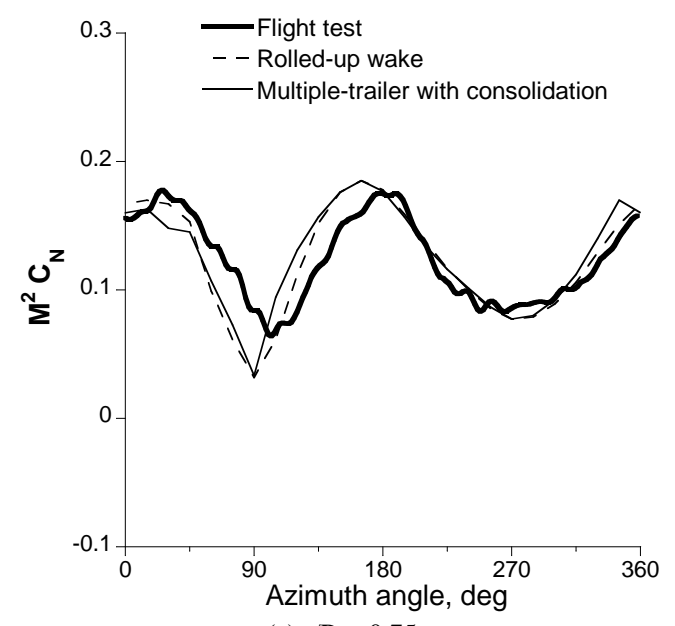

(a) $r / R=0.75$

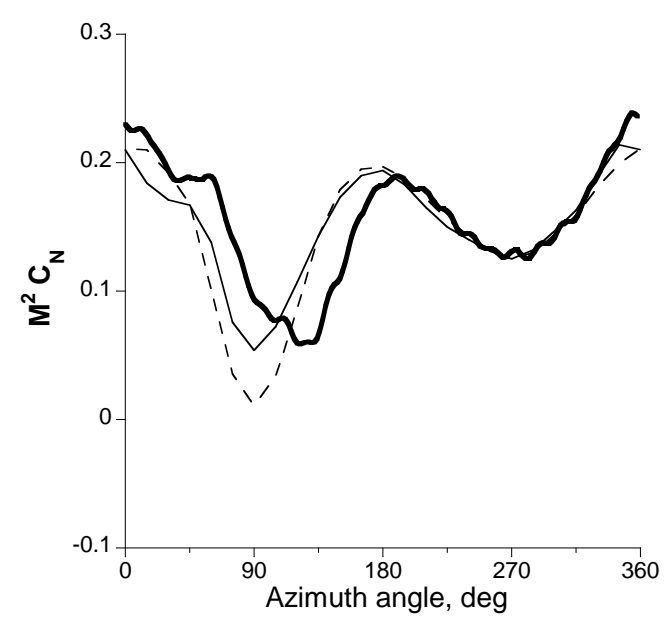

(b) $r / R=0.88$

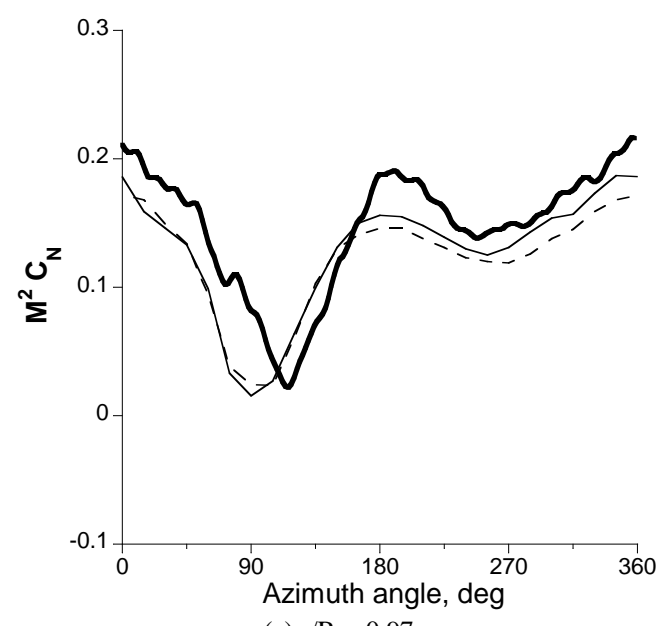

(c) $r / R=0.97$

Fig. 12: Calculated and measured blade normal force for SA $349 / 2$ at $\mu=0.361$

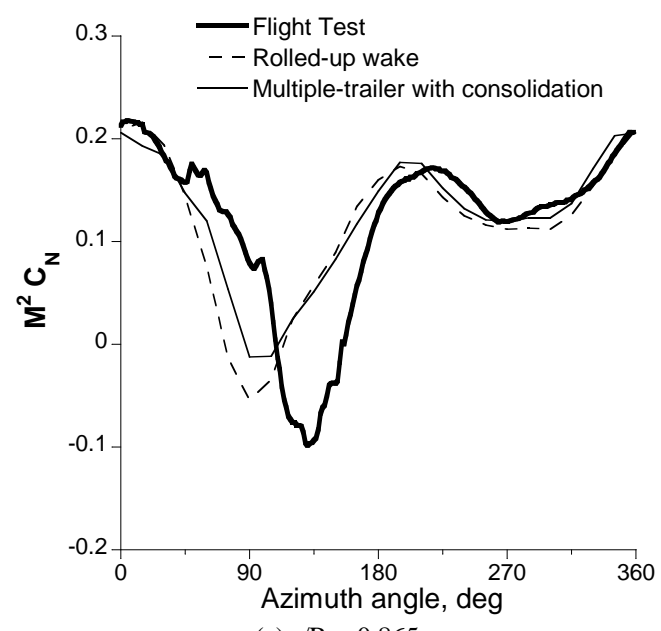

(a) $\mathrm{r} / \mathrm{R}=0.865$

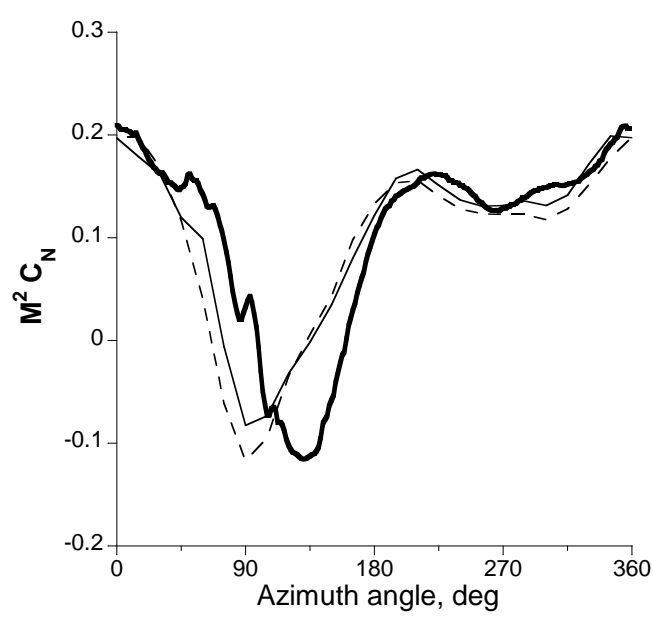

(b) $r / R=0.92$

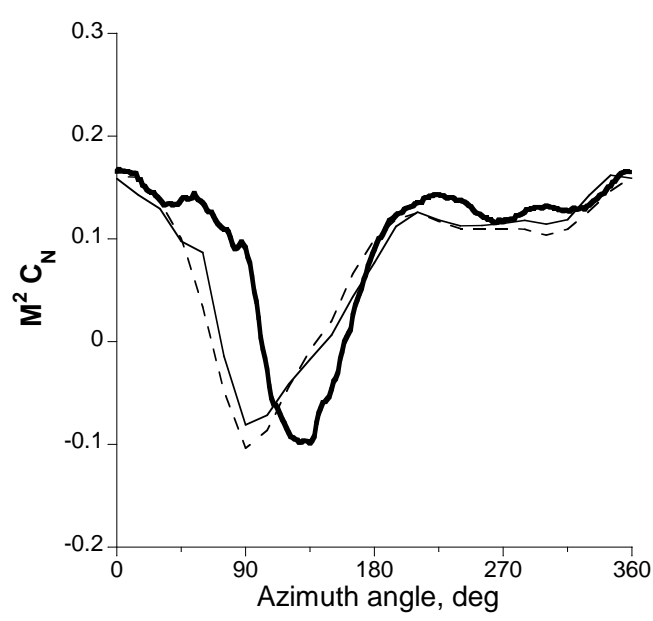

(c) $r / R=0.965$

Fig. 13: Calculated and measured blade normal force for UH-60A at $\mu=0.368$ 


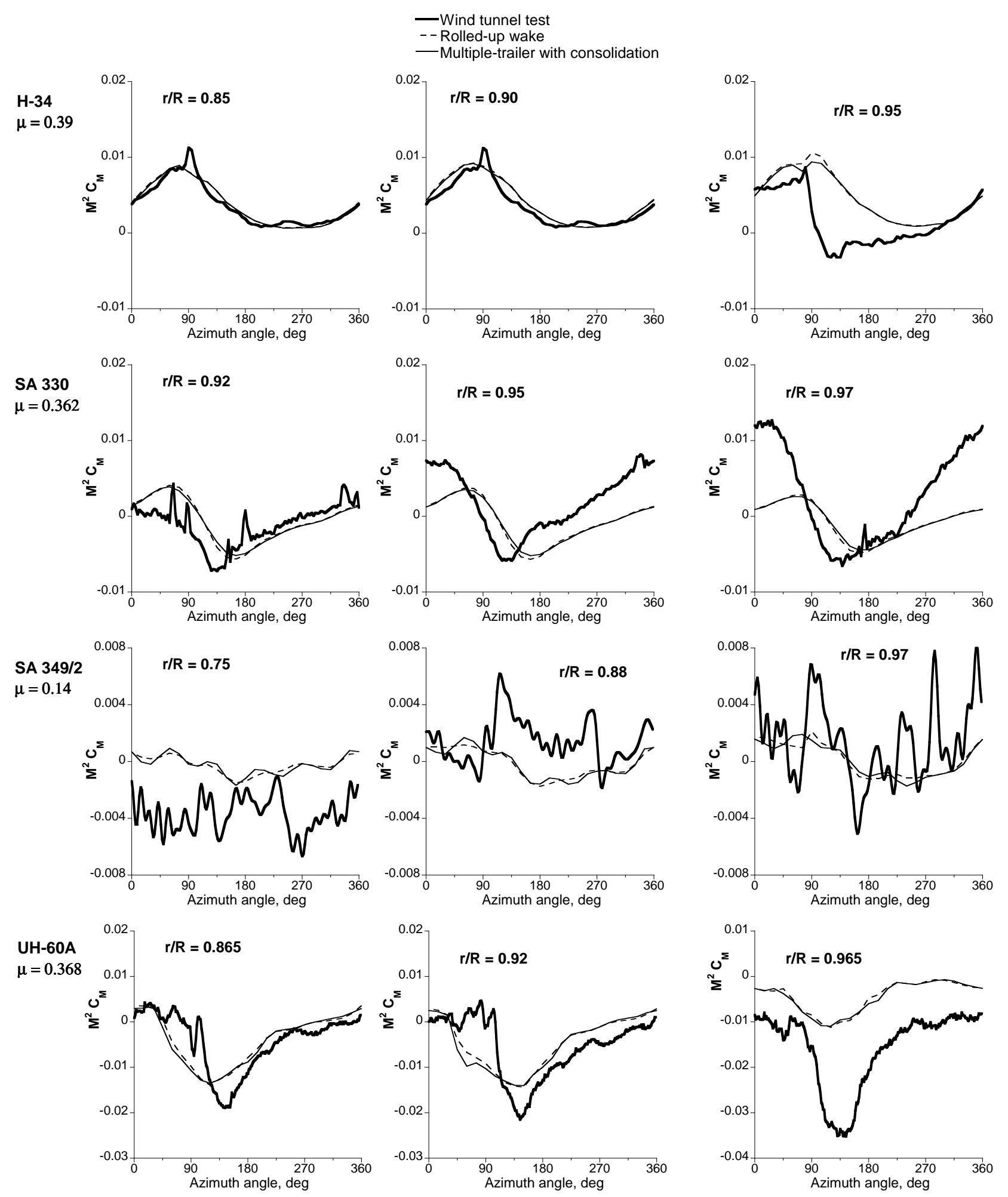

Fig. 14: Calculated and measured blade pitching moment at high speed 


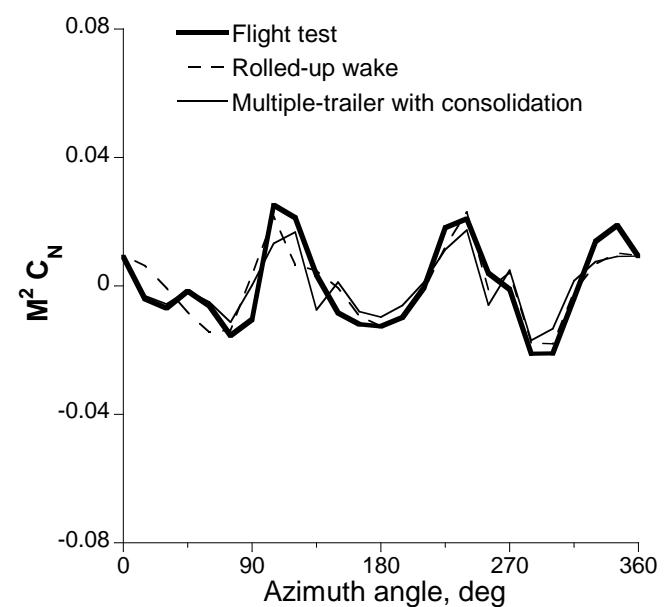

(a) $r / R=0.85$

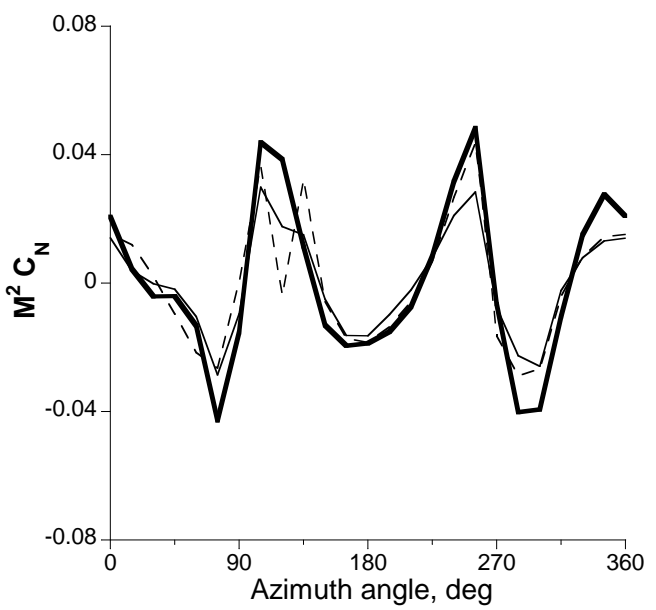

(b) $r / R=0.90$

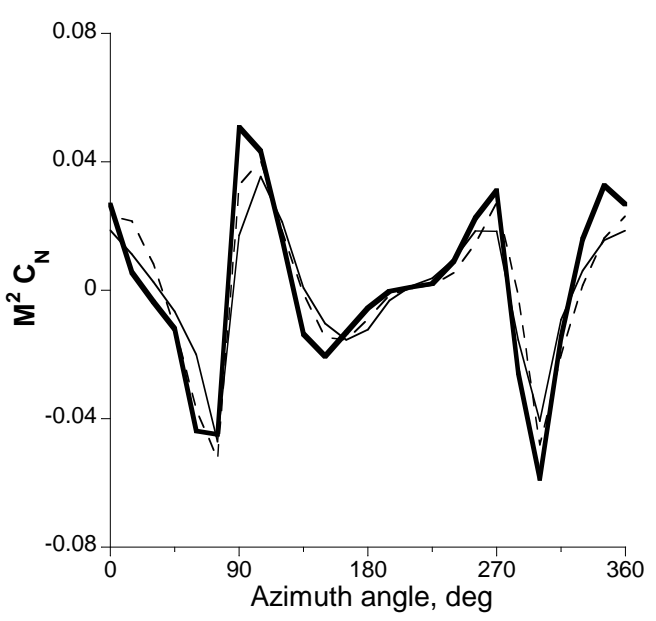

(c) $r / R=0.95$

Fig. 15: Calculated and measured blade vibratory normal force for $\mathrm{H}-34$ at $\mu=0.129$

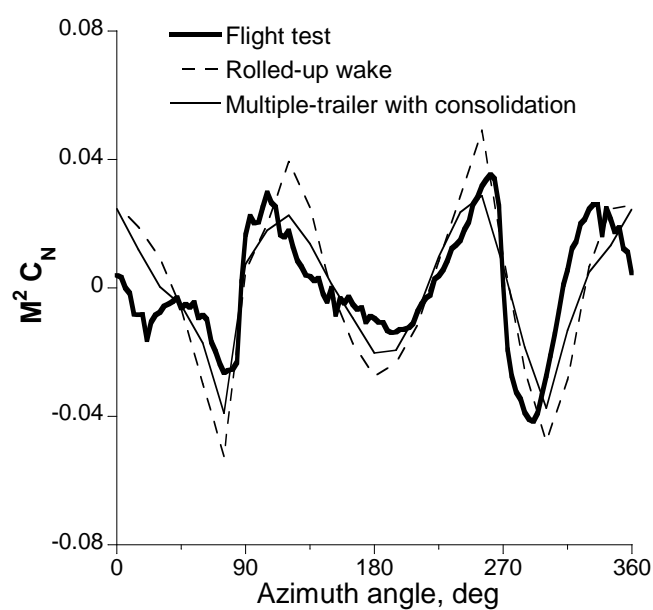

(a) $r / R=0.92$

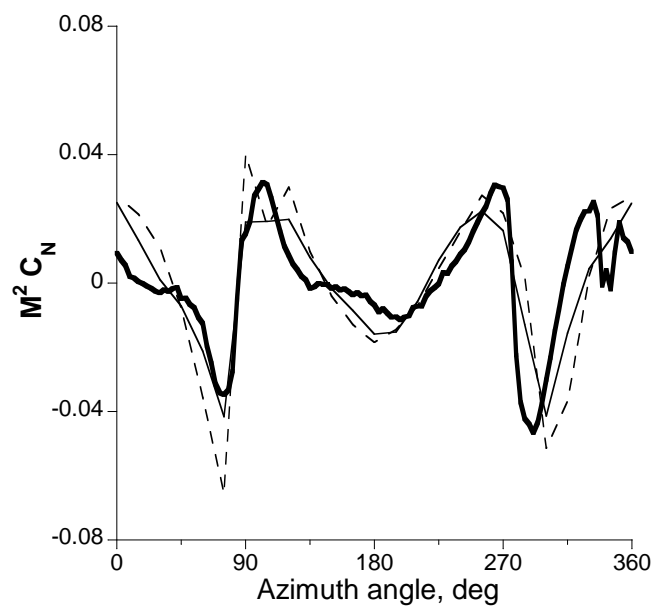

(b) $r / R=0.95$

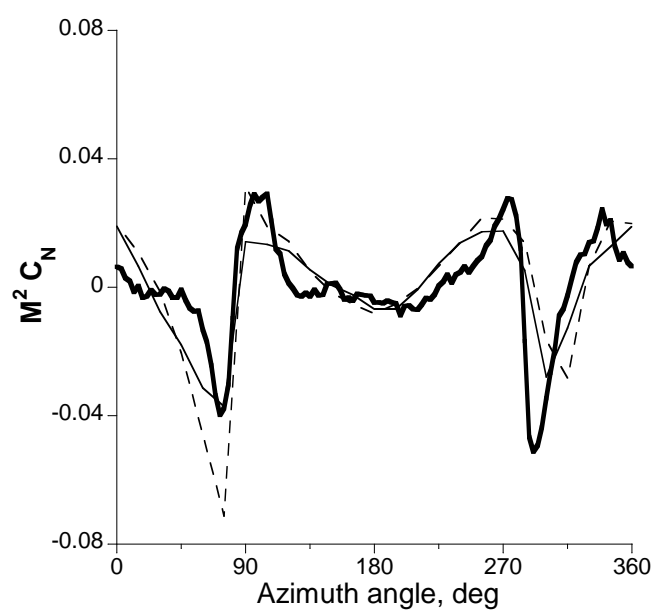

(c) $r / R=0.978$

Fig. 16: Calculated and measured blade vibratory normal force for SA 330 at $\mu=0.141$ 


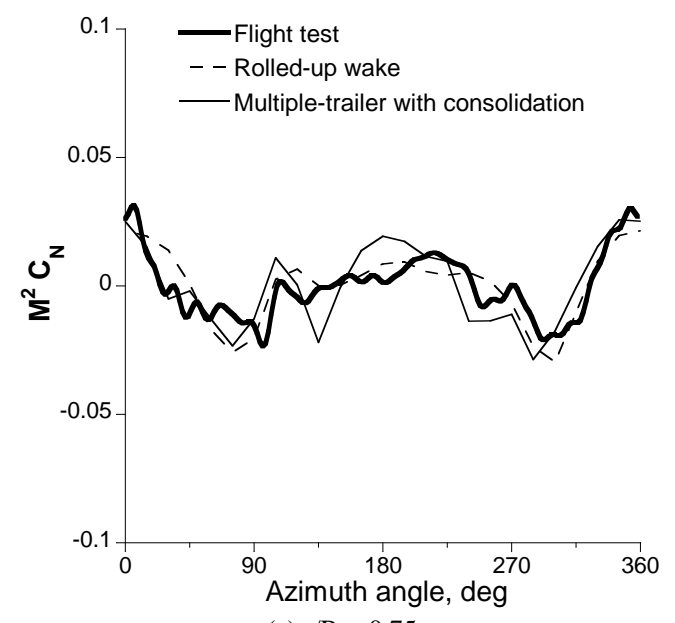

(a) $\mathrm{r} / \mathrm{R}=0.75$

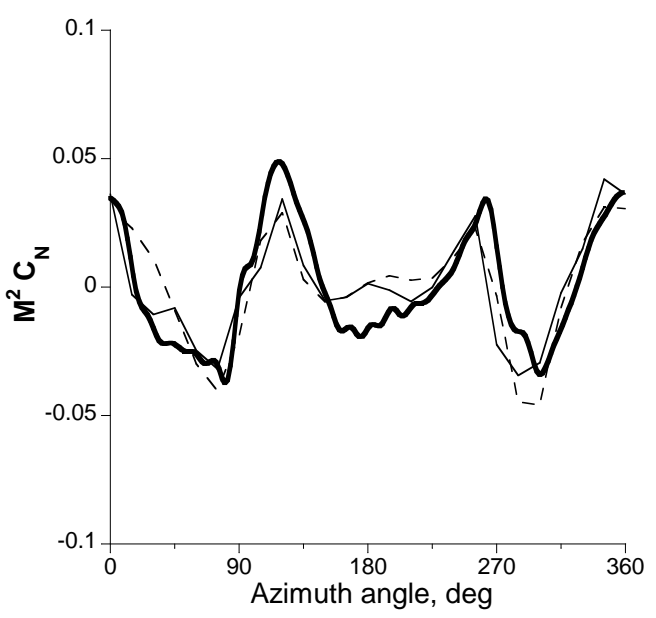

(b) $r / R=0.88$

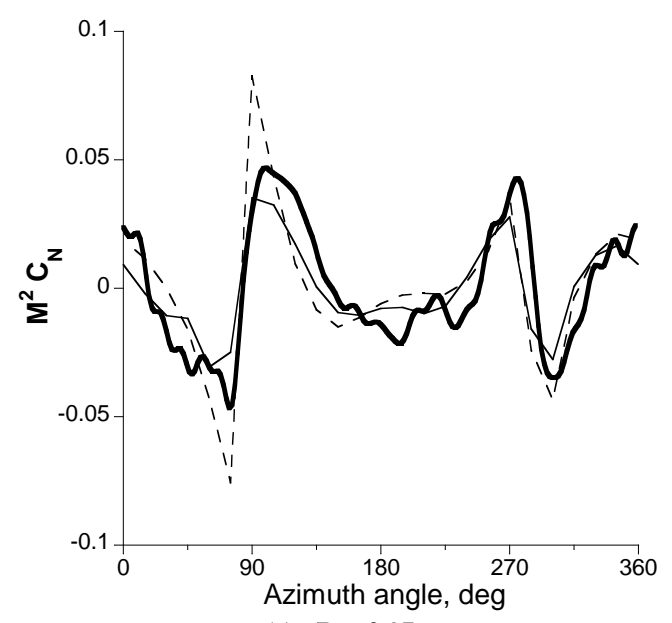

(c) $\mathrm{r} / \mathrm{R}=0.97$

Fig. 17: Calculated and measured blade vibratory normal force for SA 349/2 at $\mu=0.14$

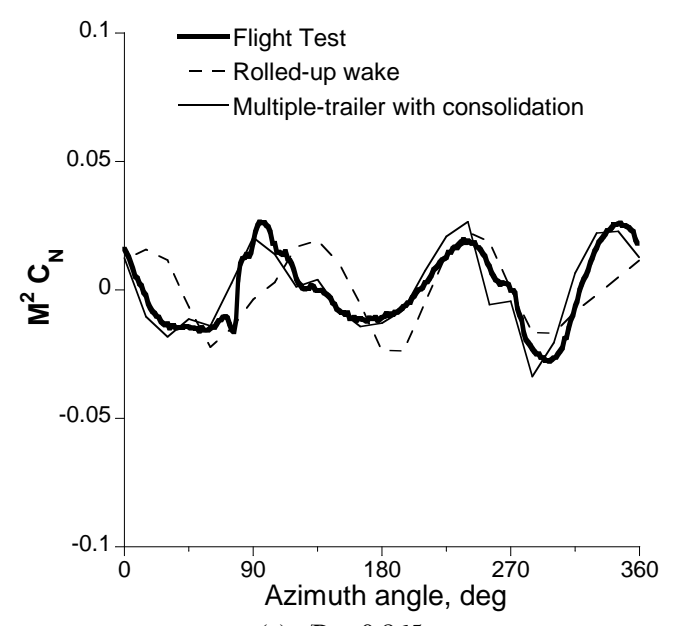

(a) $r / R=0.865$

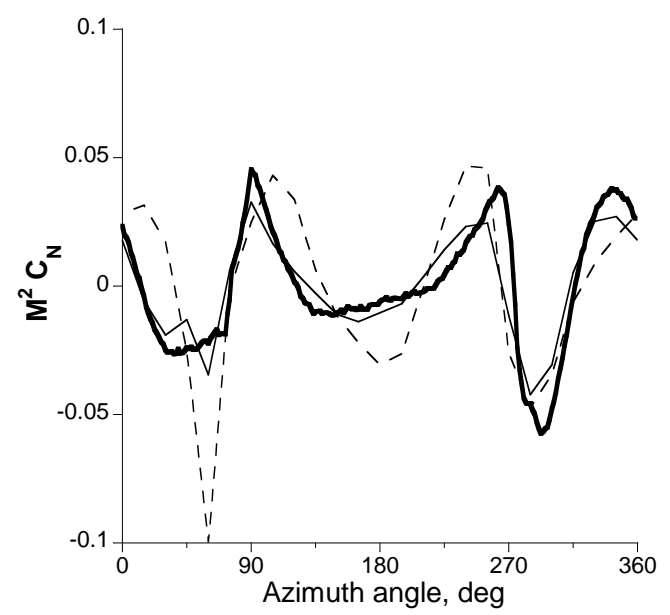

(b) $r / R=0.92$

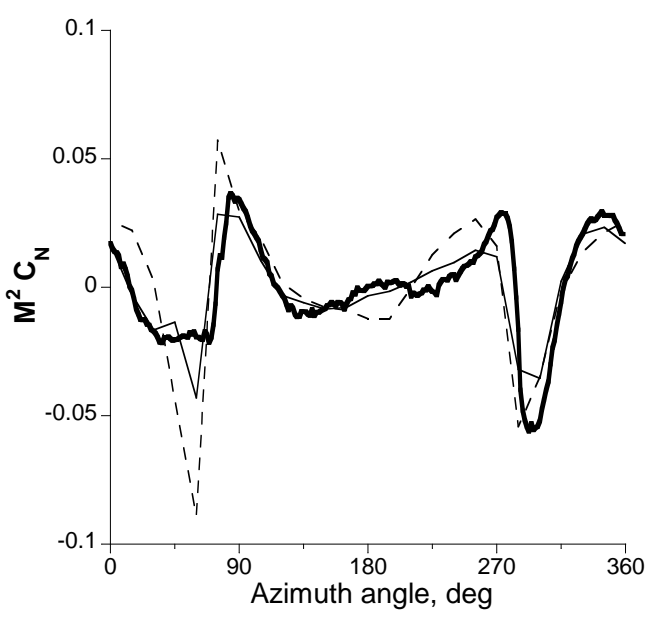

(c) $r / R=0.965$

Fig. 18: Calculated and measured blade vibratory normal force for UH-60A at $\mu=0.149$ 

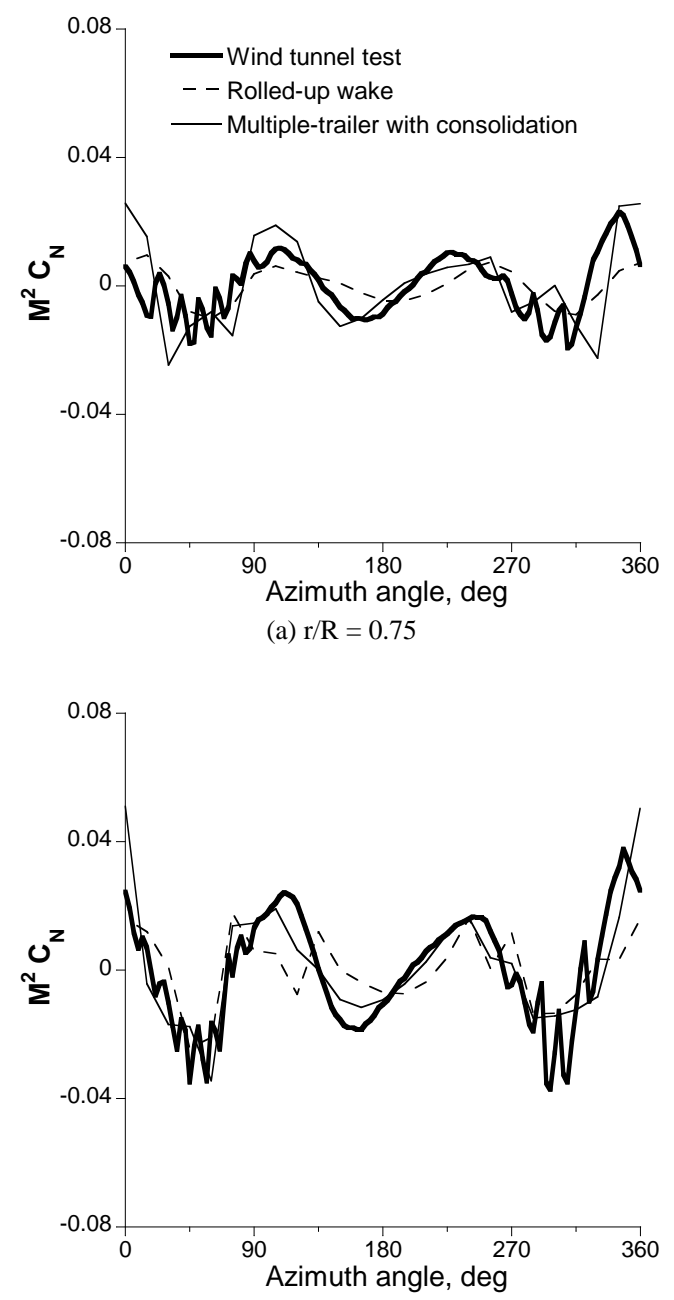

(b) $r / R=0.87$

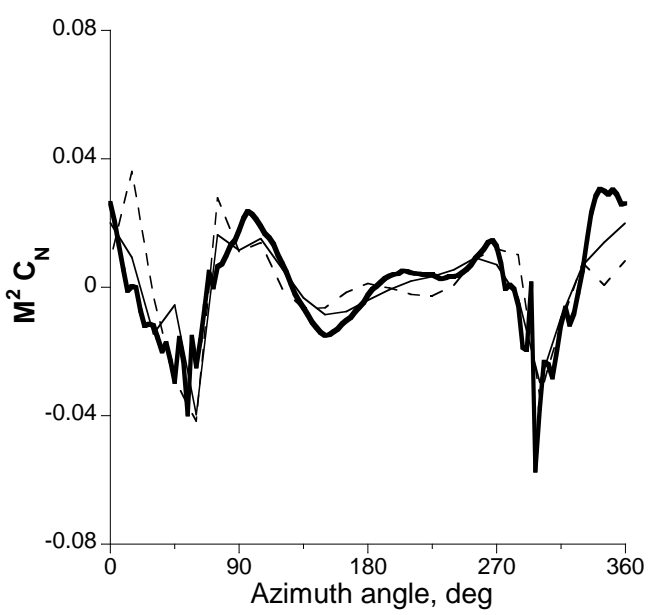

(c) $r / R=0.97$

Fig. 19: Calculated and measured blade vibratory normal force for BO-105 at $\mu=0.15$ 


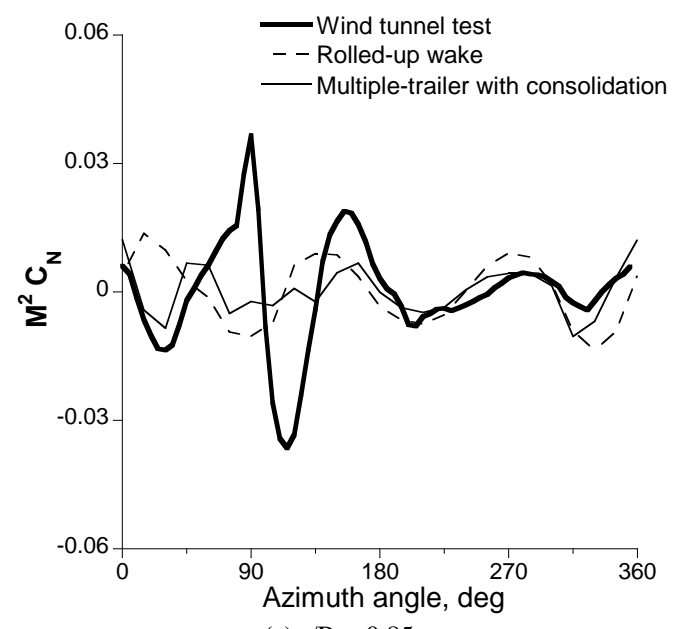

(a) $\mathrm{r} / \mathrm{R}=0.85$

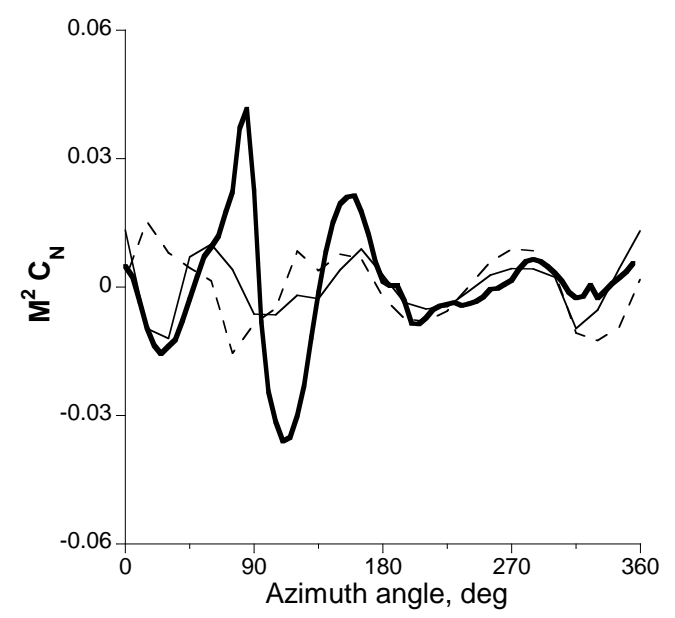

(b) $r / R=0.90$

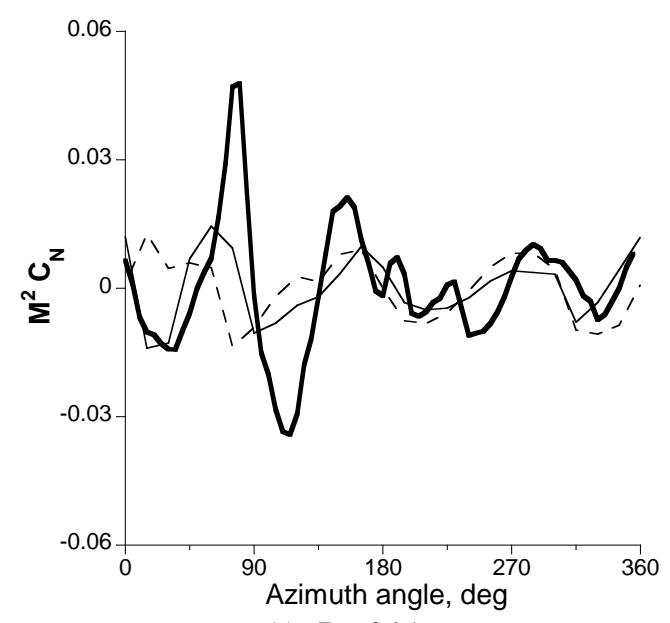

(c) $\mathrm{r} / \mathrm{R}=0.95$

Fig. 20: Calculated and measured blade vibratory normal force for $\mathrm{H}-34$ at $\mu=0.39$

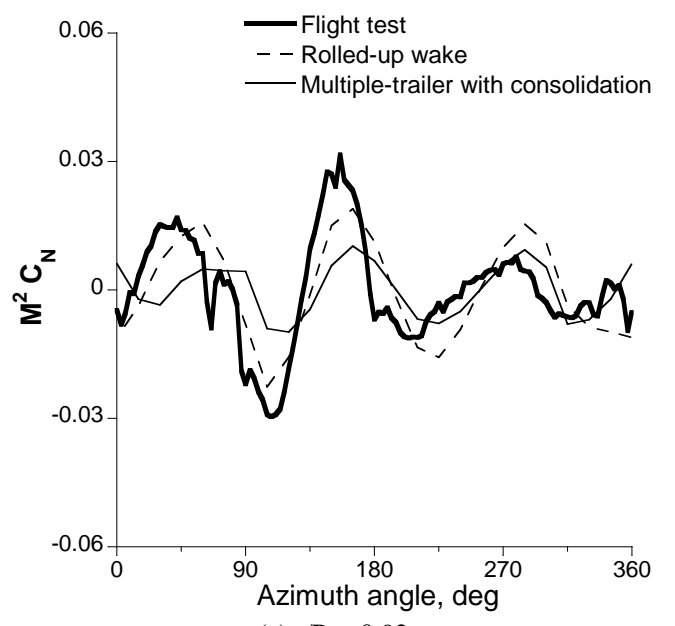

(a) $r / R=0.92$

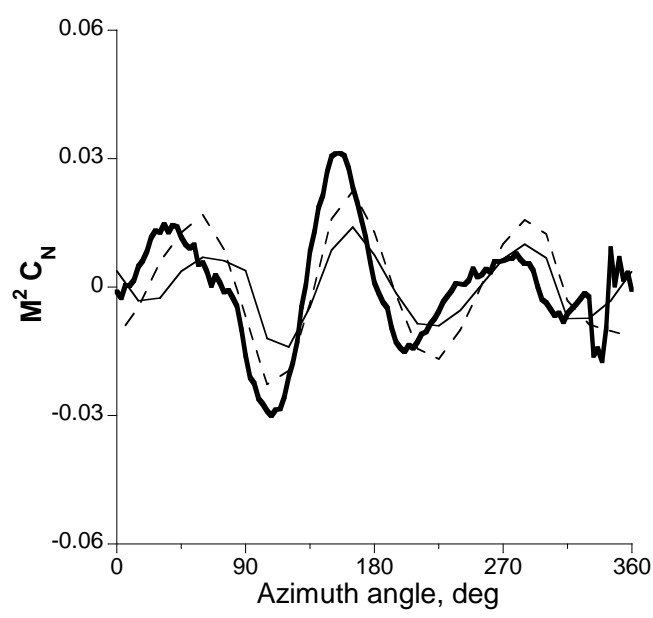

(b) $r / R=0.95$

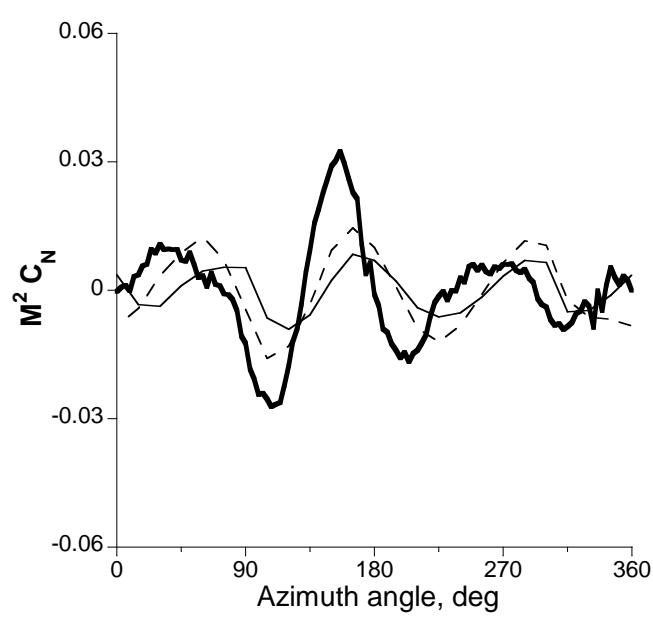

(c) $r / R=0.978$

Fig. 21: Calculated and measured blade vibratory normal force for SA 330 at $\mu=0.362$ 


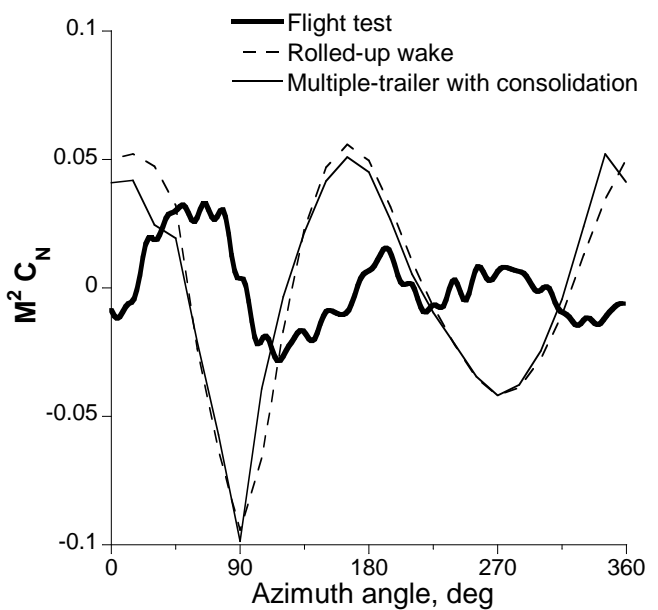

(a) $r / R=0.75$

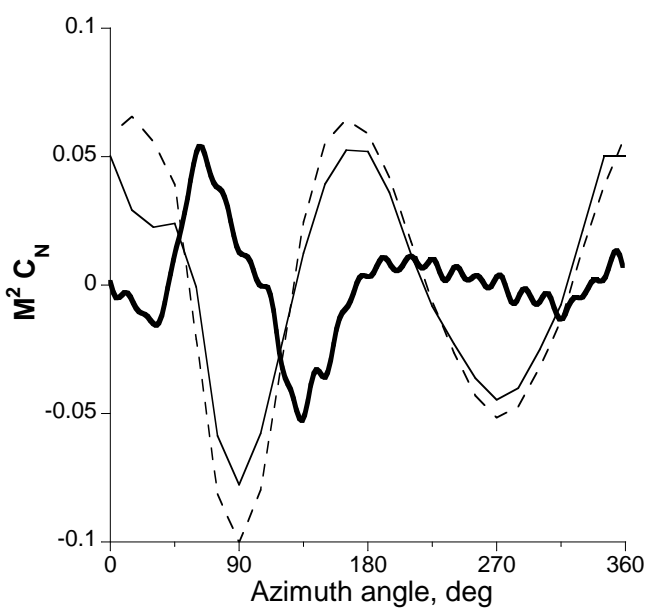

(b) $r / R=0.88$

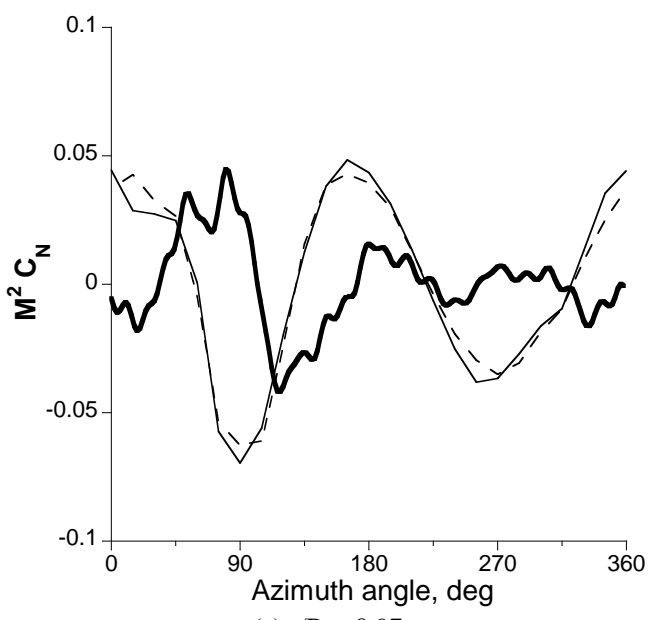

(c) $r / R=0.97$

Fig. 22: Calculated and measured blade vibratory normal force for SA $349 / 2$ at $\mu=0.361$

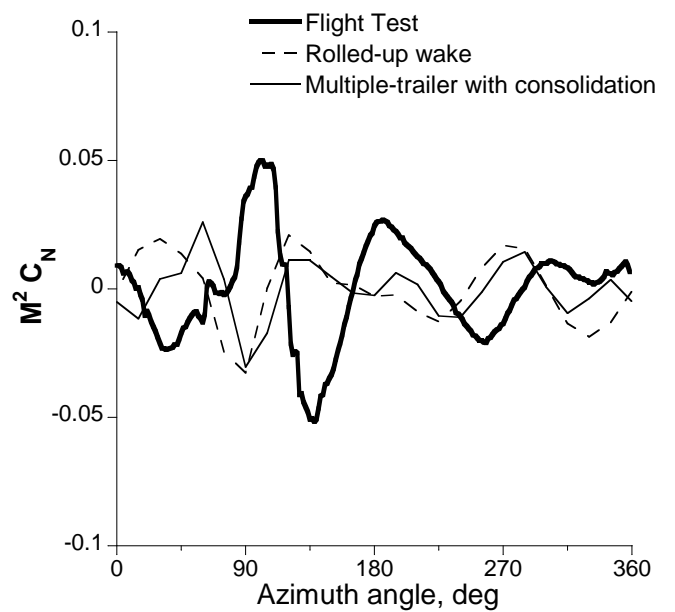

(a) $\mathrm{r} / \mathrm{R}=0.865$

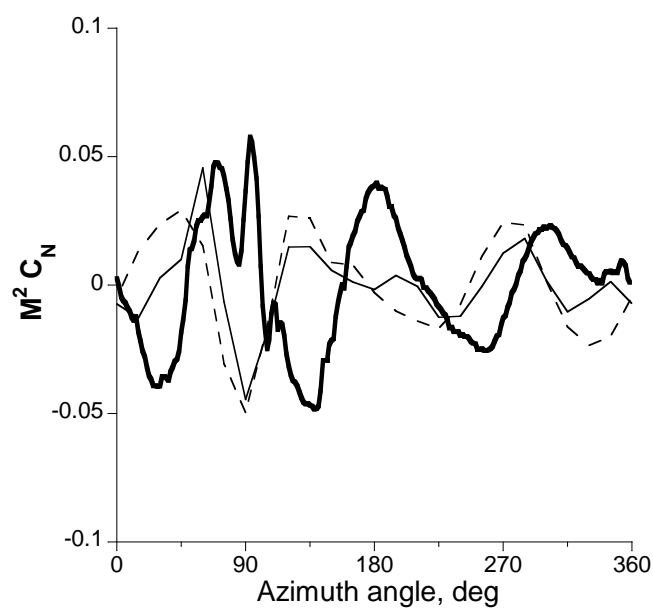

(b) $r / R=0.92$

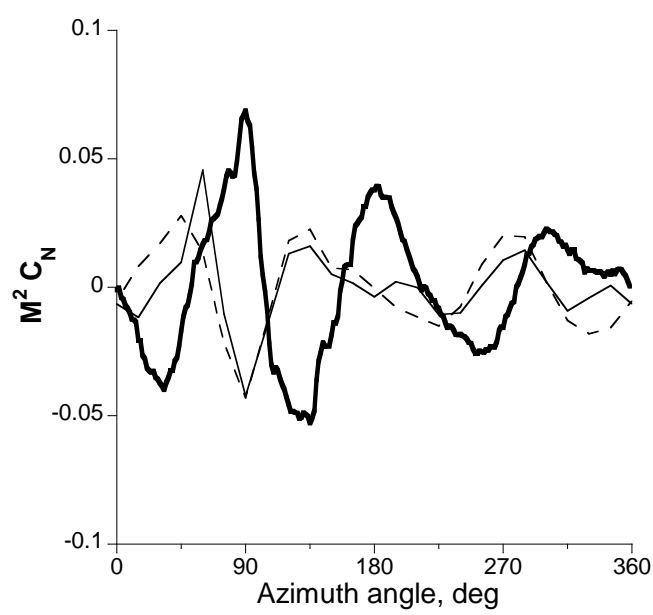

(c) $\mathrm{r} / \mathrm{R}=0.965$

Fig. 23: Calculated and measured blade vibratory normal force for UH-60A at $\mu=0.368$ 


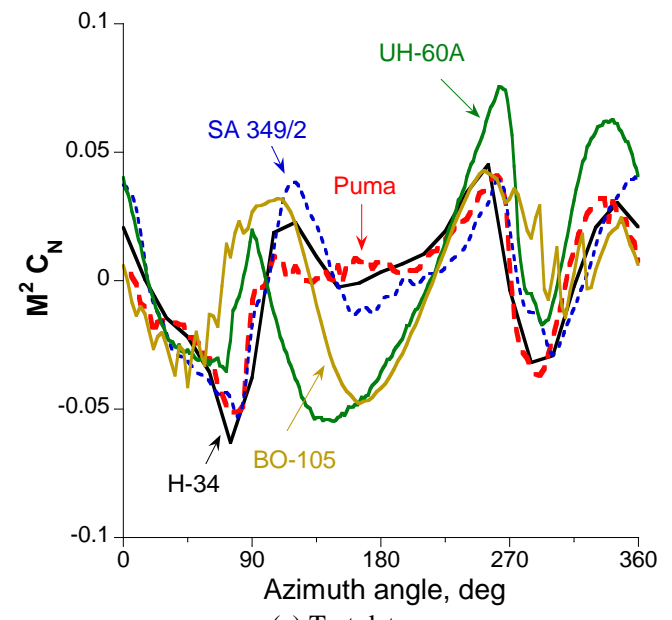

(a) Test data

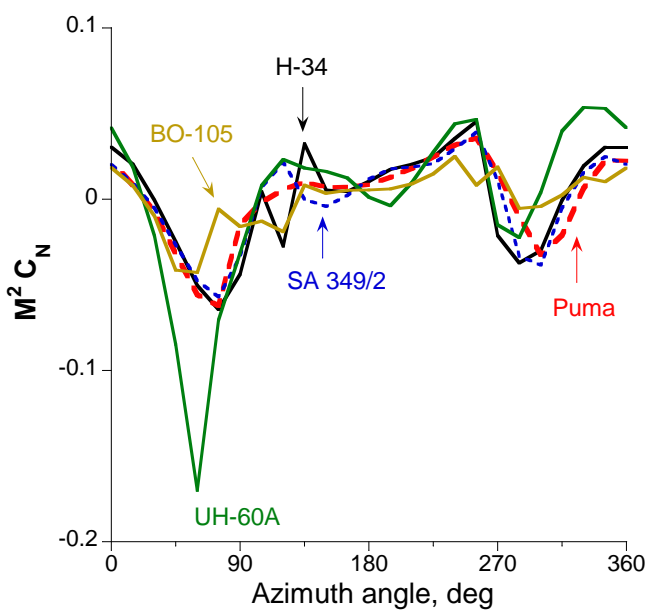

(b) Rolled-up wake

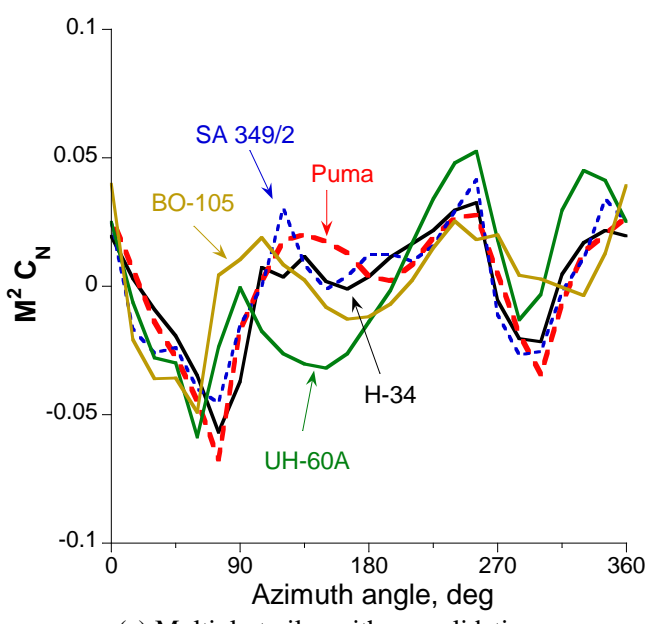

(c) Multiple-trailer with consolidation

Fig. 24: Comparison of blade oscillatory normal force at low speed near $\mathrm{r} / \mathrm{R}=0.9$

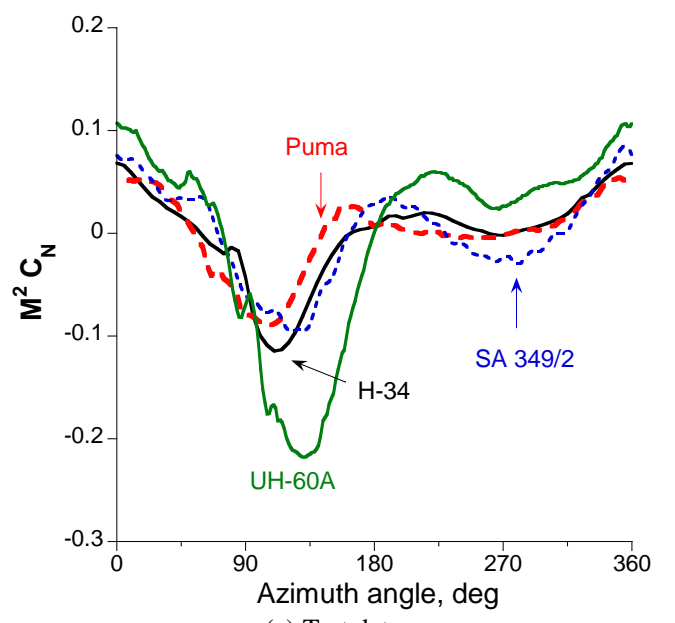

(a) Test data

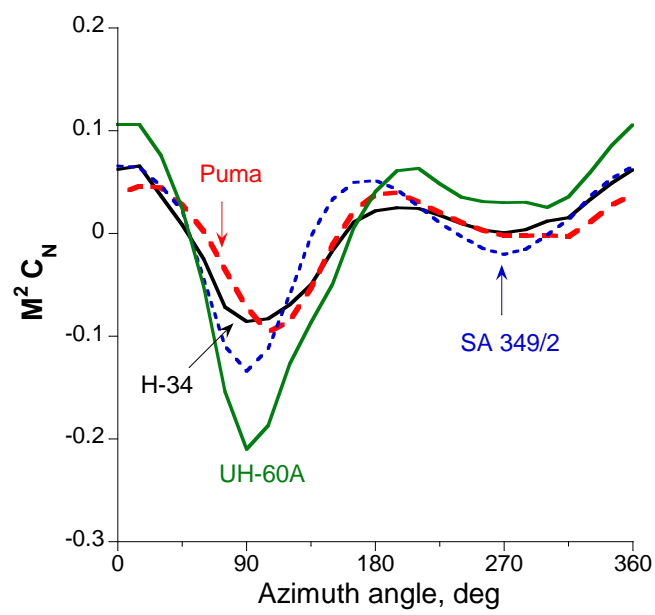

(b) Rolled-up wake

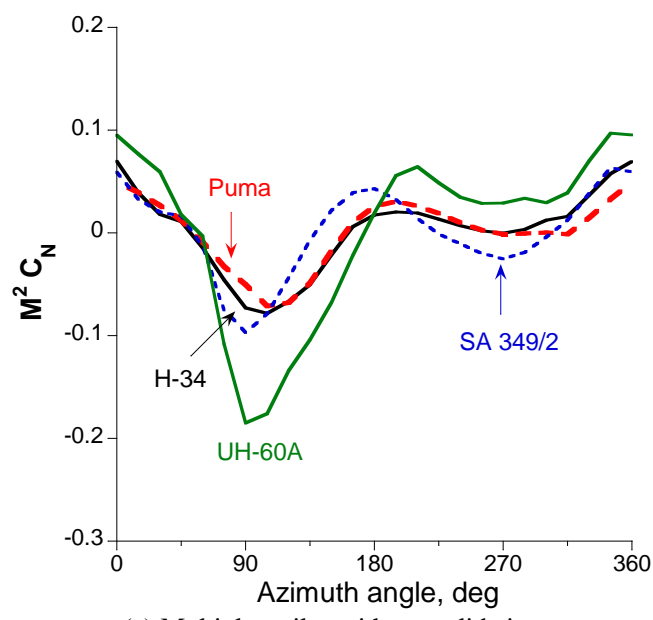

(c) Multiple-trailer with consolidation

Fig. 25: Comparison of blade oscillatory normal force at high speed near $r / R=0.9$ 


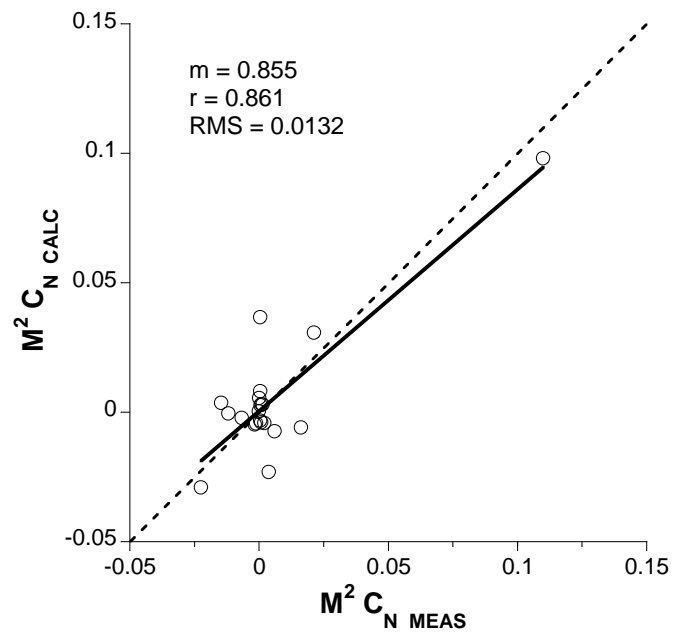

(a) Rolled-up wake

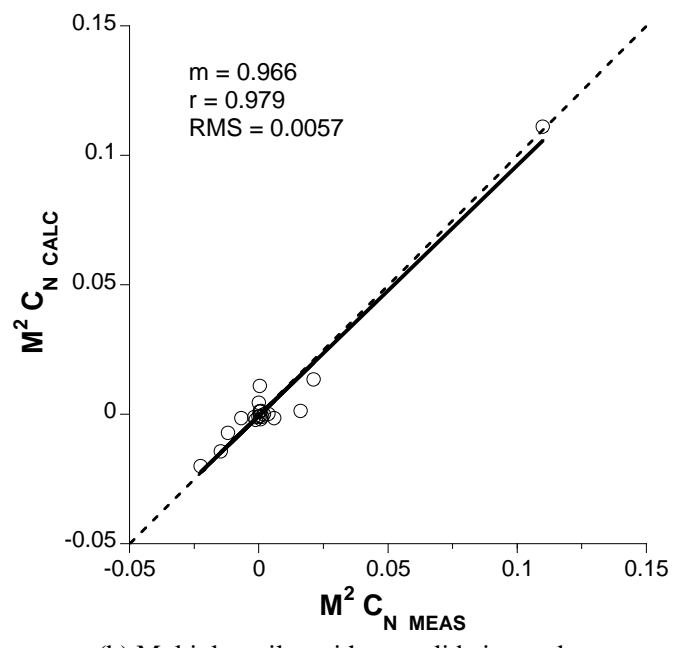

(b) Multiple-trailer with consolidation wake

Fig. 26: Harmonic correlation of blade normal force at $\mathrm{r} / \mathrm{R}=0.865$ for UH-60A at $\mu=0.149$; harmonics $0-10$
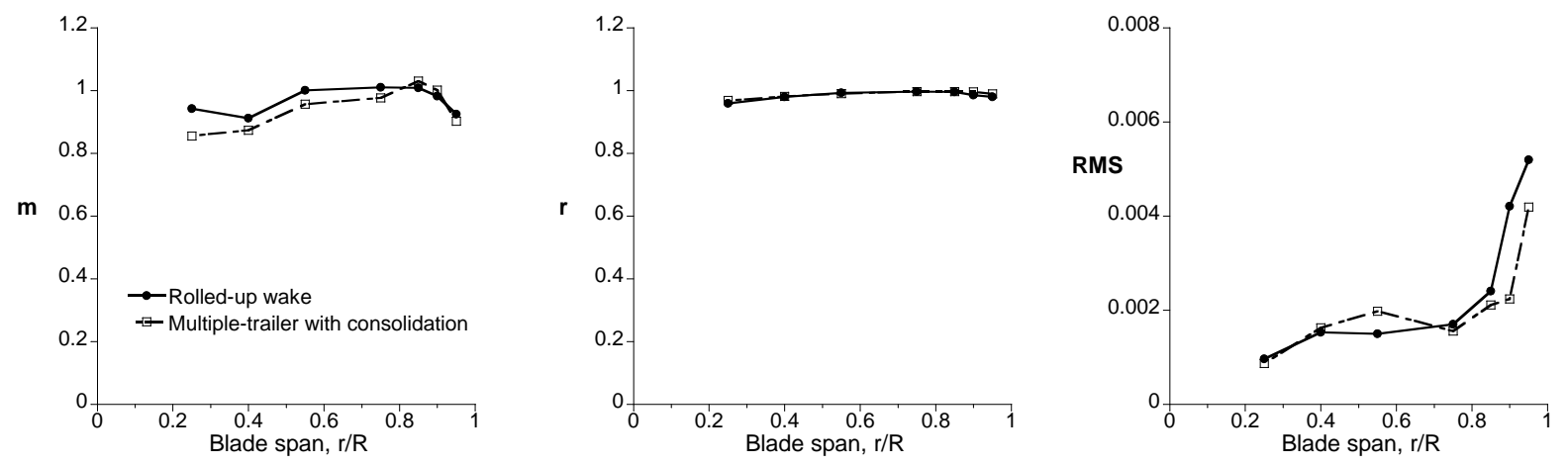

Fig. 27: Slope, correlation coefficient, and RMS error values for H-34 at $\mu=0.129$; harmonics 0-10
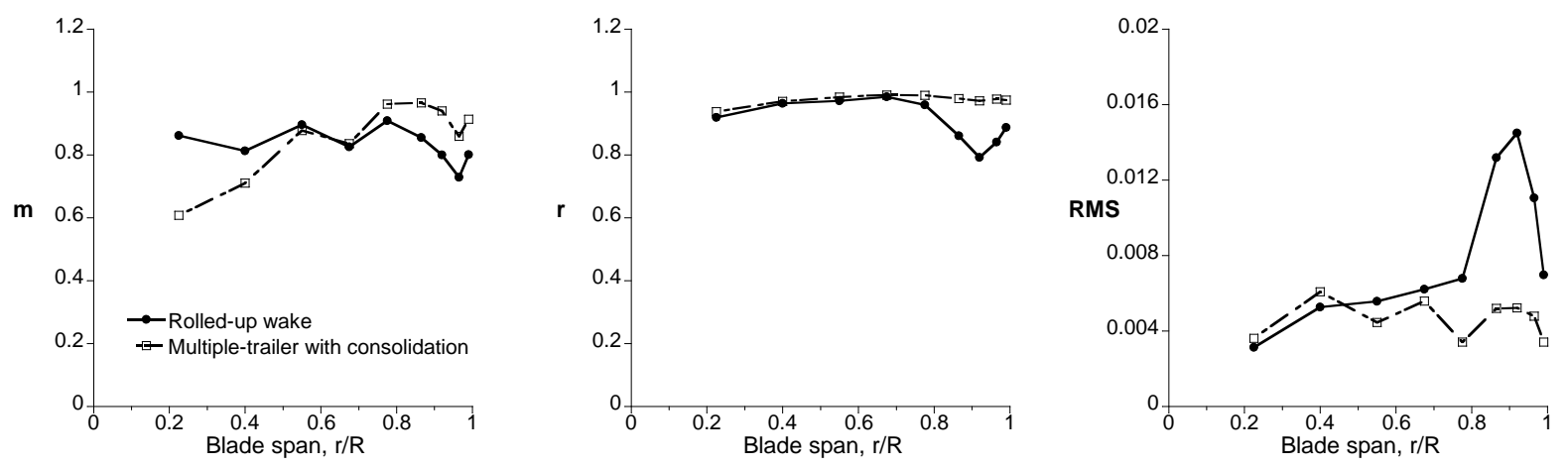

Fig. 28: Slope, correlation coefficient, and RMS error values for UH-60A at $\mu=0.149$; harmonics $0-10$ 

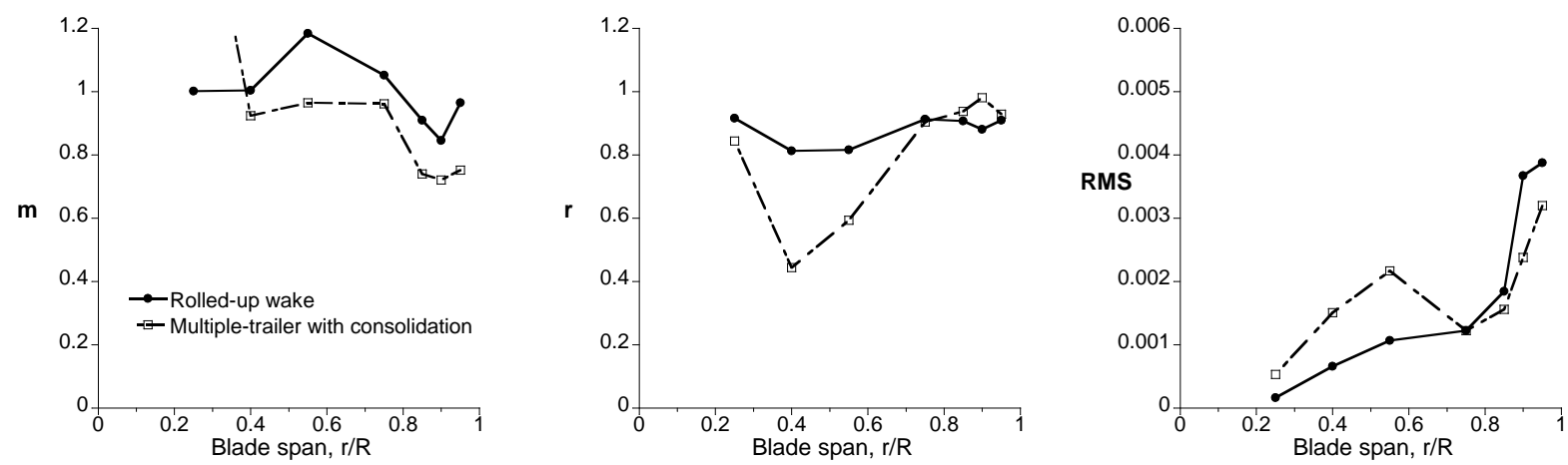

Fig. 29: Slope, correlation coefficient, and RMS error values for H-34 at $\mu=0.129$; harmonics 3-10
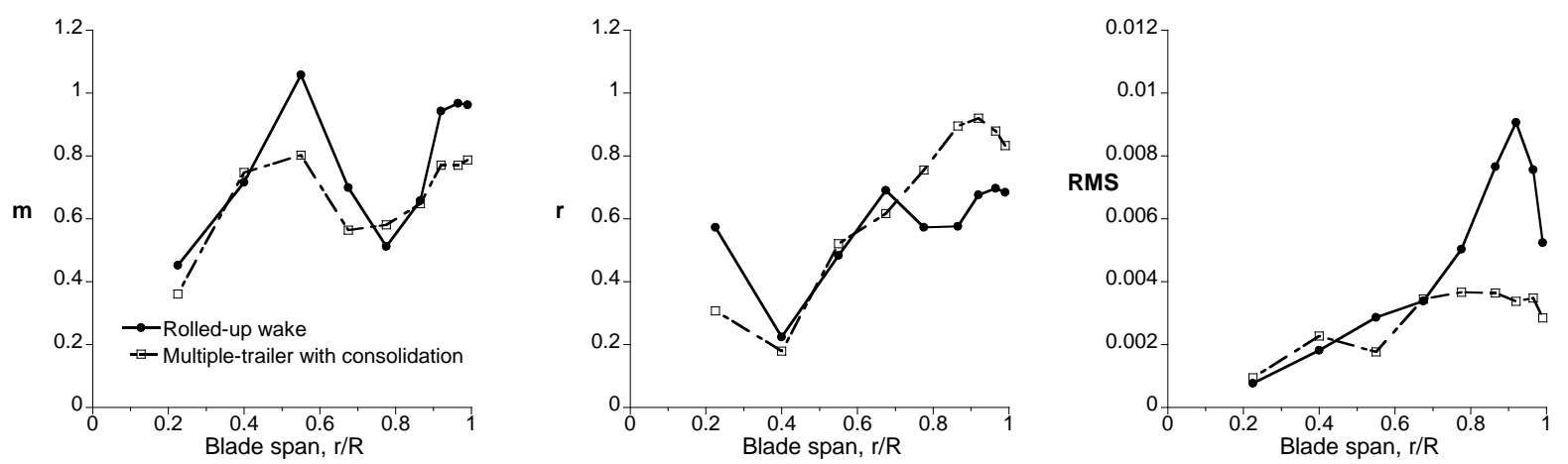

Fig. 30: Slope, correlation coefficient, and RMS error values for UH-60A at $\mu=0.149$; harmonics 3-10

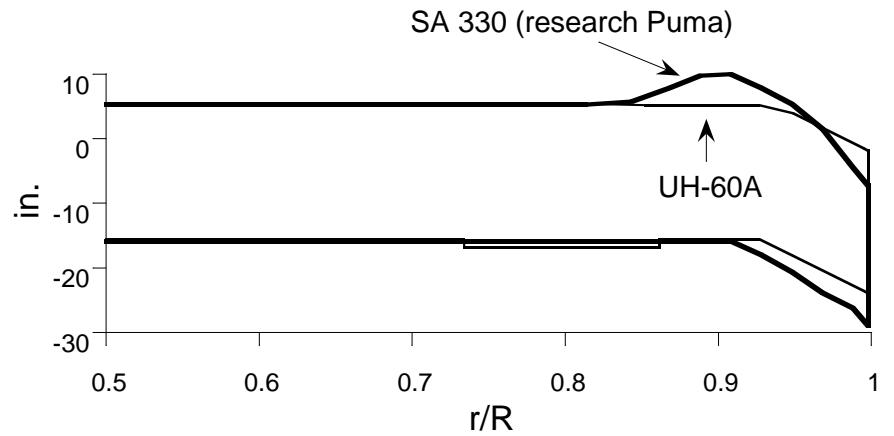

Fig. 31: Comparison of blade tip geometry between research Puma and UH-60A 

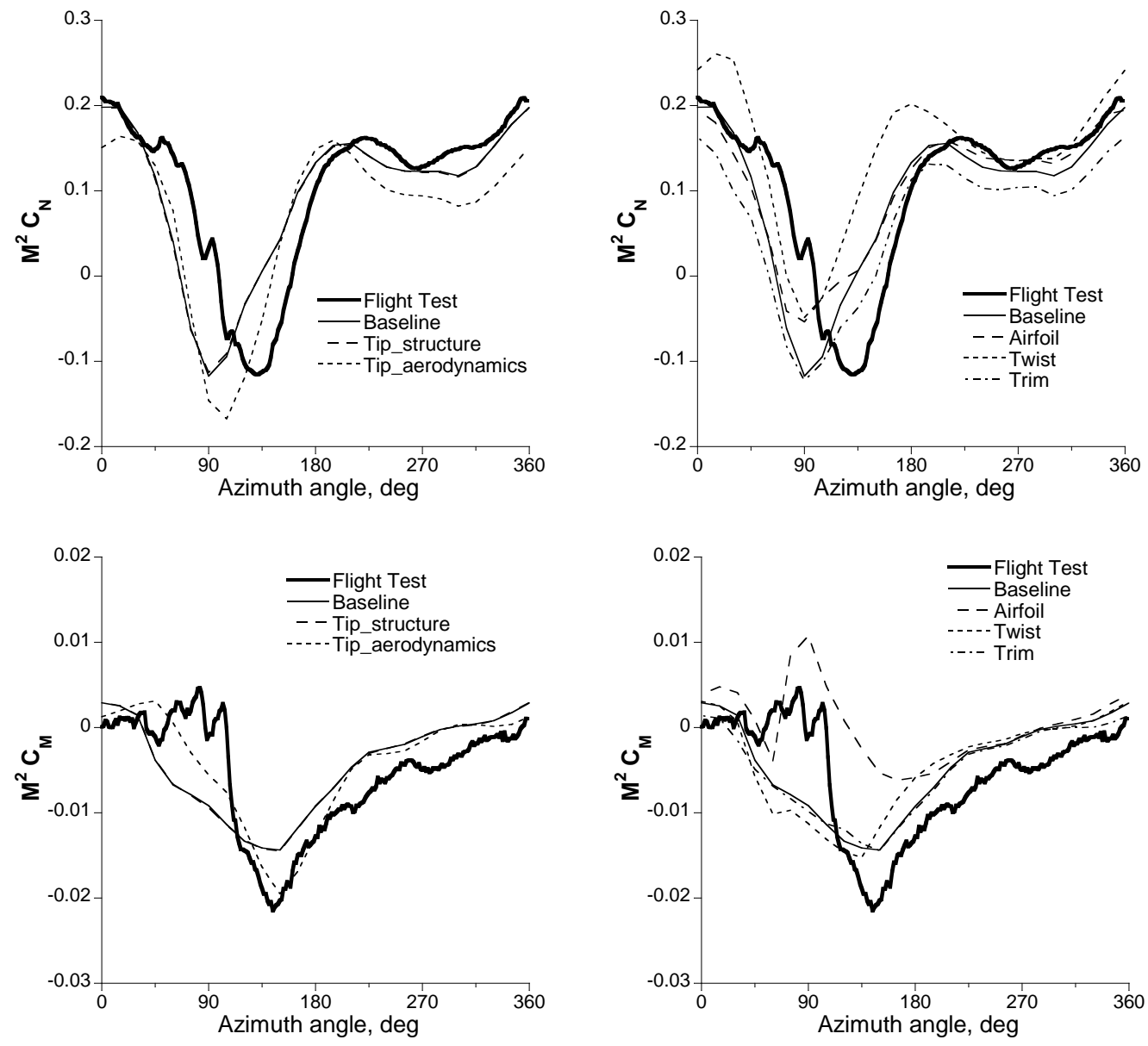

Fig. 32: Blade airloads at $r / R=0.92$ for UH-60A at $\mu=0.368$ using the Puma parameters 

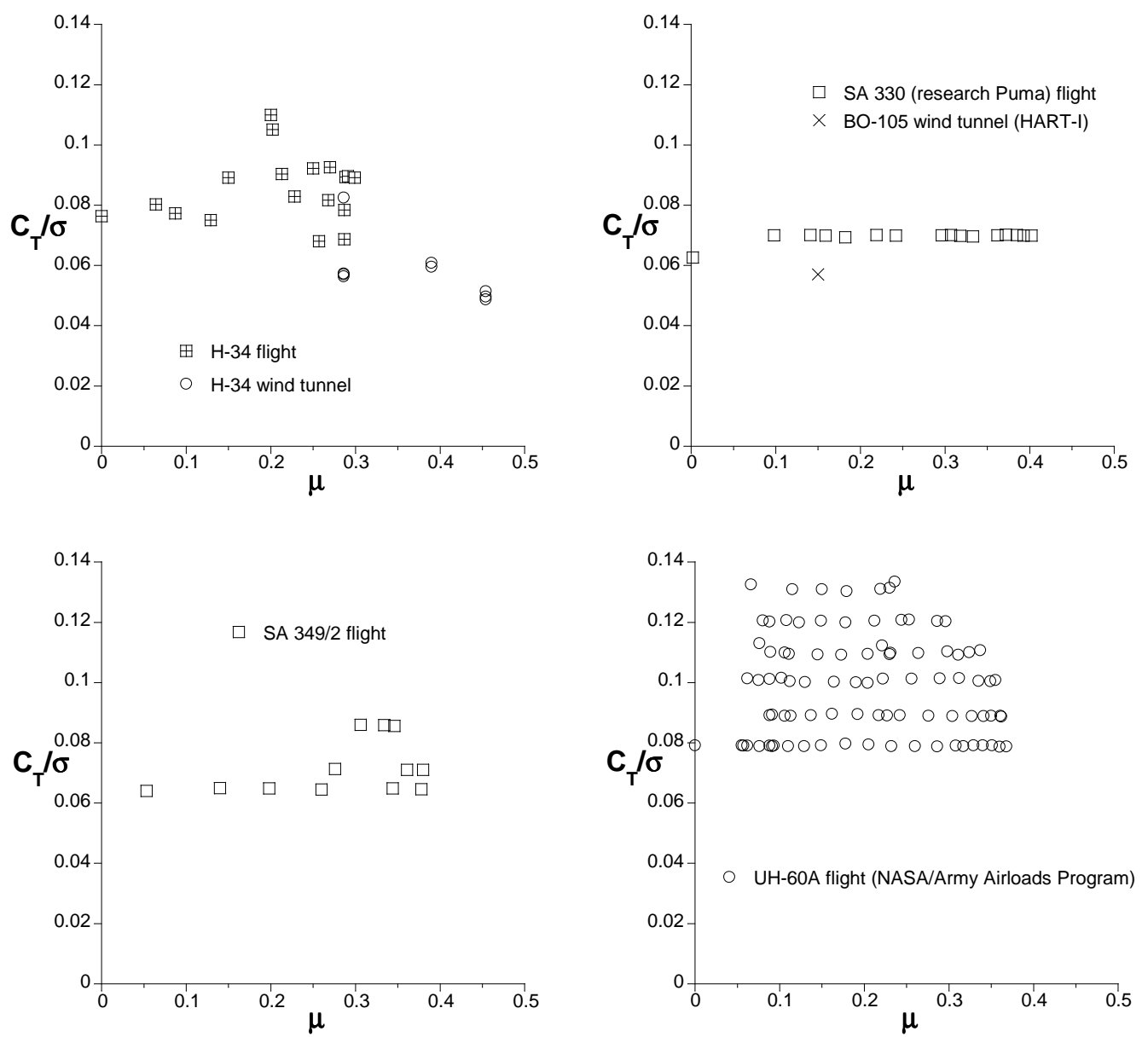

Fig. A.1: Rotor thrust coefficient and advance ratio of test data available 
Table A.1: Pressure measurement

\begin{tabular}{|c|c|c|c|}
\hline & Spanwise location (r/R) & No. of transducers & Note \\
\hline \multirow{7}{*}{ H-34 flight } & 0.25 & $5 \mathrm{D}$ & D: differential \\
\hline & 0.40 & $5 \mathrm{D}$ & U: upper surface \\
\hline & 0.55 & 7D & L: lower surface \\
\hline & 0.75 & 7D & \\
\hline & 0.85 & $11 \mathrm{D}$ & \\
\hline & 0.90 & 7D & \\
\hline & 0.95 & $7 \mathrm{D}$ & \\
\hline \multirow[t]{9}{*}{ H-34 wind tunnel } & 0.25 & 5D & \\
\hline & 0.40 & 5D & \\
\hline & 0.55 & 7D & \\
\hline & 0.75 & 7D & \\
\hline & 0.85 & 11D & \\
\hline & 0.90 & 7D & \\
\hline & 0.95 & 7D & \\
\hline & 0.97 & 4D & \\
\hline & 0.99 & $3 \mathrm{D}$ & \\
\hline \multirow[t]{3}{*}{ SA 330 flight } & 0.92 & $12 \mathrm{U}, 5 \mathrm{~L}$ & \\
\hline & 0.95 & $14 \mathrm{U}, 7 \mathrm{~L}$ & \\
\hline & 0.978 & $14 \mathrm{U}, 7 \mathrm{~L}$ & \\
\hline \multirow{3}{*}{ SA 349/2 flight } & 0.75 & $12 \mathrm{U}, 6 \mathrm{~L}$ & \\
\hline & 0.88 & $9 \mathrm{U}, 6 \mathrm{~L}$ & \\
\hline & 0.97 & $11 \mathrm{U}, 7 \mathrm{~L}$ & \\
\hline \multirow{9}{*}{ UH-60A flight } & 0.225 & $10 \mathrm{U}, 10 \mathrm{~L}$ & \\
\hline & 0.40 & $10 \mathrm{U}, 10 \mathrm{~L}$ & \\
\hline & 0.55 & $10 \mathrm{U}, 10 \mathrm{~L}$ & \\
\hline & 0.675 & $10 \mathrm{U}, 10 \mathrm{~L}$ & \\
\hline & 0.775 & $12 \mathrm{U}, 12 \mathrm{~L}$ & \\
\hline & 0.865 & $15 \mathrm{U}, 13 \mathrm{~L}$ & \\
\hline & 0.92 & $15 \mathrm{U}, 14 \mathrm{~L}$ & \\
\hline & 0.965 & $15 \mathrm{U}, 15 \mathrm{~L}$ & \\
\hline & 0.99 & $15 \mathrm{U}, 15 \mathrm{~L}$ & \\
\hline \multirow[t]{3}{*}{ BO-105 wind tunnel } & 0.75 & $27 \mathrm{U}, 17 \mathrm{~L}$ & \\
\hline & 0.87 & $14 \mathrm{U}, 10 \mathrm{~L}$ & \\
\hline & 0.97 & $27 \mathrm{U}, 17 \mathrm{~L}$ & \\
\hline
\end{tabular}

